\title{
Revealing the selective mechanisms of inhibitors to PARP-1 and PARP-2 via multiple computational methods
}

\author{
Hongye Hu ${ }^{1}$, Buran Chen ${ }^{2}$, Danni Zheng ${ }^{2}$, Guanli Huang ${ }^{\text {Corresp. } 1}$ \\ ${ }^{1}$ Department of Thyroid and Breast Surgery, The First Affiliated Hospital of Wenzhou Medical University, Wenzhou, China \\ 2 The First Clinical Medical College, Wenzhou Medical University, Wenzhou, China \\ Corresponding Author: Guanli Huang \\ Email address: huangguanli@wzhospital.cn
}

Background. Research has shown that Poly-ADP-ribose polymerases 1 (PARP-1) is a potential therapeutic target in the clinical treatment of breast cancer. An increasing number of studies have focused on the development of highly selective inhibitors that target PARP-1 over PARP-2, its closest isoform, to mitigate potential side effects. However, due to the highly conserved and similar binding sites of PARP-1 and PARP-2, there is a huge challenge for the discovery and design of PARP-1 inhibitors. Recently, it was reported that a potent PARP-1 inhibitor named NMS-P118 exhibited greater selectivity to PARP-1 over PARP-2 compared with a previously reported drug Niraparib. However, the mechanisms underlying the effect of this inhibitor remains unclear.

Methods. In the present study, classical molecular dynamics (MD) simulations and accelerated molecular dynamics (aMD) simulations combined with structural and energetic analysis were used to investigate the structural dynamics and selective mechanisms of PARP-1 and PARP-2 that are bound to NMS-P118 and Niraparib with distinct selectivity.

Results. The results from classical MD simulations indicated that the selectivity of inhibitors may be controlled by electrostatic interactions, which were mainly due to the residues of GIn-322, Ser-328, Glu-335, and Tyr-455 in helix $\alpha \mathrm{F}$. The energetic differences were corroborated by the results from aMD simulations.

Conclusion. This study provides new insights about how inhibitors specifically bind to PARP-1 over PARP-2, which may help facilitate the design of highly selective PARP-1 inhibitors in the future. 
1 Revealing the selective mechanisms of inhibitors to PARP-1 and PARP-2 via

2 multiple computational methods

4 Hongye $\mathrm{Hu}^{1}$, Buran $\mathrm{Chen}^{2}$, Danni Zheng ${ }^{2}$, Guanli Huang1,*

$6{ }^{1}$ Department of Thyroid and Breast Surgery, The First Affiliated Hospital of Wenzhou Medical

7 University, Wenzhou 325000, Zhejiang, China

$8 \quad 2$ The First Clinical Medical College, Wenzhou Medical University, Wenzhou 325000, Zhejiang,

9 China

10

11 * Correspondence

12 Guanli Huang

13 Department of Department of Thyroid and Breast Surgery, The First Affiliated Hospital of 14 Wenzhou Medical University, Shangcai Village, Nanbaixiang Town, Ouhai District, Wenzhou 15 325000, Zhejiang, China

16 E-mail: huangguanli@wzhospital.cn; Tel: 86-0577 55578166 
25 Abstract

Background. Research has shown that Poly-ADP-ribose polymerases 1 (PARP-1) is a potential therapeutic target in the clinical treatment of breast cancer. An increasing number of studies have focused on the development of highly selective inhibitors that target PARP-1 over PARP-2, its closest isoform, to mitigate potential side effects. However, due to the highly conserved and similar binding sites of PARP-1 and PARP-2, there is a huge challenge for the discovery and design of PARP-1 inhibitors. Recently, it was reported that a potent PARP-1 inhibitor named NMS-P118 exhibited greater selectivity to PARP-1 over PARP-2 compared with a previously reported drug Niraparib. However, the mechanisms underlying the effect of this inhibitor remains unclear.

Methods. In the present study, classical molecular dynamics (MD) simulations and accelerated molecular dynamics (aMD) simulations combined with structural and energetic analysis were used to investigate the structural dynamics and selective mechanisms of PARP-1 and PARP-2 that are bound to NMS-P118 and Niraparib with distinct selectivity.

Results. The results from classical MD simulations indicated that the selectivity of inhibitors may be controlled by electrostatic interactions, which were mainly due to the residues of Gln-322, Ser328, Glu-335, and Tyr-455 in helix $\alpha \mathrm{F}$. The energetic differences were corroborated by the results from aMD simulations.

Conclusion. This study provides new insights about how inhibitors specifically bind to PARP-1 over PARP-2, which may help facilitate the design of highly selective PARP-1 inhibitors in the future.

Keywords: PARP-1; PARP-2; Selective mechanisms; Molecular dynamics simulations 


\section{Introduction}

As one of the most commonly diagnosed malignancies in women, breast cancer accounts for about a quarter of all female cancer cases (Siegel et al. 2018). In the past few years, the incidence of breast cancer has continued to rise, with more than 1 million new cases worldwide each year (Siegel et al. 2018). It is estimated that a total of 5-10\% of all breast cancer cases are genetically susceptible to the disease, with multiple breast cancer susceptibility genes having been proposed, including breast cancer 1 (BRCA1) and breast cancer 2 (BRCA2), two of the major genes (Begg et al. 2008). Currently, sequencing of these two genes is considered as the optimal approach to determining the mutation status in breast cancer patients (Pfeffer et al. 2017). Previous studies have shown that homologous recombination-deficient tumor cells resulting from BRCA1 or BRCA2 gene mutations are hypersensitive to the inhibitory effect of poly-ADP ribose polymerase1 (PARP-1) (Lin \& Kraus 2017; McCann 2019). One mechanism that has been proposed as a tentative explanation is that inhibition of PARP-1 blocks DNA single strand break repair and leads to the formation of unrepaired double strand breaks at the replication fork (Kim et al. 2020; Min \& Im 2020; Wang et al. 2019b). Aside from DNA damage repair, PARP-1 is also involved in a wide variety of cellular processes, such as cell proliferation and cell death. The implication of PARP-1 in these processes is a result of the diverse substrates in PARP-1, such as nuclear proteins, which are involved in apoptotic cell death, cell cycle regulation, chromatin decondensation, inflammation, and transcriptional regulation (Jubin et al. 2016). Due to these functions, PARP-1 inhibitors have been developed as the first class of cancer therapeutics in clinical trials (Min \& Im 2020).

Currently, four PARP-1 drugs have been approved by the U.S. Food and Drug Administration (FDA), including olaparib, niraparib, talazoparib, and rucaparib. In addition to these commercially available PARP-1 drugs, numerous PARP-1 inhibitors have also entered different phases of clinical research, targeting multiple types of tumor either collectively or as single agents (Min \& Im 2020). Most of the marketed PARP-1 drugs and inhibitors exhibit poor selectivity when 
targeting PAPR-1. For instance, olaparib, the first PAPR-1 drug approved by FDA, also interacts with PAPR-1 close homologues PARP-2 and PARP-3 (Gunderson \& Moore 2015; Min \& Im 2020). Similarly, Niraparib, Talazoparib and rucaparib were also found to exhibit no selectivity between PARP-1 and PARP-2 (Min \& Im 2020). There is ample evidence in previous studies that inhibition of PARP-2 could produce potentially undesirable side effects (Farres et al. 2013; Navarro et al. 2017). For instance, Farrés et al. reported that loss of PARP-2 leads to a shortened red blood cell lifespan and impaired differentiation of erythroid progenitor cells, thereby causing chronic anemia (Farres et al. 2013). In light of this situation, a great deal of efforts has been put into the design and development of potent PARP-1inhibitors with high selectivity, especially between PARP-1 and PARP-2 (Eltze et al. 2008; Fatima et al. 2014; Papeo et al. 2015). However, given high sequence similarity (84\% identity and 90\% similarity) and conserved catalytic domain (Figure 1A-C), increasing the selectivity of inhibitors remains a huge challenge (Yelamos et al. 2011).

Up to now, some potent inhibitors with high selectivity to PARP-1 over PARP-2 have been discovered, such as WD2000-012547, BYK204165 and NMS-P118 (Eltze et al. 2008; Fatima et al. 2014; Papeo et al. 2015). Despite these promising results, little computational research has been conducted to elucidate the selective mechanisms underlying the effect of these inhibitors. To this end, two representative inhibitors (Niraparib, NMS-P118) with divergent selectivity to PARP-1 and PARP-2 were utilized in this study to demonstrate such mechanisms (Ison et al. 2018; Papeo et al. 2015). Niraparib (also formerly known as MK-4827) is a novel, highly selective, and orally available PARP-1 and PARP-2 small molecule drug developed by Tesaro, approved by FDA in 2017 to treat ovarian cancer (Ison et al. 2018). Recent clinical studies show that the combined use of niraparib and pembrolizumab can have promising antitumor effects on patients with advanced or metastatic triple-negative breast cancer (Lin et al. 2019). NMS-P118, another inhibitor originally designed by Papeo et al., has been shown to exhibit $\sim 150$-fold selectivity to PAPR-1 over PARP2 (Figure 1D-E). In addition, the researchers also reported the co-crystal structure of PARP1 and PARP-2 bound to NMS-P118, and proposed that helix $\alpha \mathrm{F}$ in the two proteins may be 
103

104

105

106

107

108

109

110

111

112

113

114

115

116

117

118

119

120

121

122

123

124

125

126

127

128

129

responsible for the drug selectivity, as the induced binding pocket is larger in PARP-1 than in PARP-2 (Papeo et al. 2015). However, this explanation seems far from satisfactory. Here, classical molecular dynamics (cMD) simulations and accelerated molecular dynamics (aMD) simulations were employed to clarify the selective mechanisms between PARP-1 and PARP-2 using two representative inhibitors (Niraparib, NMS-P118). A combination of classical MD simulations, the root-mean-square deviations (RMSD), principal component analysis (PCA), dynamical crosscorrelation (DCC) analysis, and root-mean-square fluctuations (RMSF) was applied to investigate the effects of the inhibitors on the protein flexibility and dynamic behavior of key parts of PARP-1 and PARP-2. Afterwards, binding free energy calculations and per-residue free energy decompositions based on the molecular mechanics/generalized Born solvent area (MM/GBSA) method were performed to highlight key residues related to selectivity. Next, aMD simulations combined with RMSD, PCA, DCC and free energy landscape (FEL) analyses were carried out to examine in detail the local energy minima and the conformational space that were not illuminated in the classical MD simulations. Overall, these results can be effective to deepen our understanding of the selective mechanisms between PARP-1 and PARP-2, and may help facilitate the design of novel inhibitors to improve drug selectivity.

9

\section{Methods and materials}

\section{Preparation of the initial systems}

The three-dimensional structures of human PARP-1 bound to NMS-P118 (PDB ID: 5A00), Niraparib (PDB ID: 4R6E), and PARP-2 bound to NMS-P118 (PDB ID: 4ZZY) were obtained from the Protein Data Bank (Papeo et al. 2015; Thorsell et al. 2017). The Loops/Refine Structure module of UCSF Chimera program was employed to model the missing side-chains and loop structures (Pettersen et al. 2004). The PDB2PQR Server was employed to estimate the protonation states of ionizable side chains (Dolinsky et al. 2004). The initial coordinates of PARP-2 bound to Niraparib were constructed using the AutoDock program (Morris et al. 2009). The grid size of cubic box, which was centered on the binding pocket, was set to $60 \times 60 \times 60$ xyz points with a 
130

131

132

133

134

135

136

137

138

139

140

141

142

143

144

145

146

147

148

149

150

151

152

153

154

155

156

grid spacing of $0.375 \AA$. The AutoDockTools program was employed to assign the Gasteiger partial charges to PARP-2 and Niraparib. The affinity maps of grids were estimated using AutoGrid program. The docking protocol involved the generation of 200 conformations. The maximum number of energy evaluations and iterations were set to $25,000,000$ and 3,000, respectively. Other parameters were set to default. The top-ranked structure was used for the subsequent molecular dynamics (MD) simulation analyses.

\section{Classical MD simulation}

The prepared crystal structures of PARP-1 bound to NMS-P118 and Niraparib, PARP-2 bound to NMS-P118, and modeled complex of PARP-2 bound to Niraparib were applied to determine the dynamic structural behavior via Assisted Model Building with Energy Refinement 18 (Amber 18) program. The restrained electrostatic potential (RESP) fitting technique was employed to estimate the partial atomic charges of NMS-P118 and Niraparib (Wang et al. 2000). The parameters of protein and ligand were derived from the ff14SB force field the General Amber Force Field 2 (GAFF2) in Amber 18 (Maier et al. 2015; Vassetti et al. 2019). Each of the prepared complexes was solvated in a cubic box containing TIP3P water molecules, with a minimum distance of $15 \AA$ from any edge of the box to any complex atom. Counter ions of an appropriate quantity were added to the system to preserve overall charge neutrality.

Prior to the classical MD simulation, two-step minimizations, heating and equilibration were performed. At first, two-step minimizations were undertaken to eliminate bad contacts between the solvent molecules and the complexes. To reduce the counterions and water molecules to a minimum, a harmonic constraint of $20 \mathrm{kcal} \cdot \mathrm{mol}^{-1} \cdot \AA^{-2}$ was first imposed on the four complexes. Then, restriction was eliminated in order for all atoms to move freely. During each stage, the steepest descent minimization of 7,000 steps was performed, followed by conjugate gradient minimization of 7,000 steps. Thereafter, Langevin thermostat with a position restraint of 20 $\mathrm{kcal} \cdot \mathrm{mol}^{-1} \cdot \AA^{-2}$ was applied to gradually heat up each complex from $0 \mathrm{~K}$ to $300 \mathrm{~K}$ over $300 \mathrm{ps}$. Then, each complex was equilibrated at $300 \mathrm{~K}$ with $1000 \mathrm{ps}$ simulation time in the isothermal 
157

158

159

160

161

162

163

164

165

166

167

168

169

170

171

172

173

174

175

$$
\begin{array}{ll}
\Delta V(r)=0 & \Delta V(r) \geq E \\
\Delta V(r)=\frac{[E-\Delta V(r)]^{2}}{\alpha+[E-\Delta V(r)]} & \Delta V(r)<E
\end{array}
$$

176

177

178

179

180

where $\alpha$ is the acceleration factor that governs the size of the boost. The $\alpha$ is calculated with equations (3) and (4), and the $E$ is calculated according to equations (5) and (6). The boost parameters $E$ and $\alpha$ for the total boost $\left(E_{\text {total }}\right.$ and $\left.\alpha_{\text {total }}\right)$ and dihedral boost $\left(E_{\text {dihedral }}\right.$ and $\left.\alpha_{\text {dihedral; }}\right)$ are based on the corresponding average $E_{\text {total }}\left(V_{\text {total_avg }}\right)$ and average $E_{\text {dihedral }}\left(V_{\text {dihedral_avg }}\right)$, 
181 which are calculated from the classical MD simulations prior to the aMD simulations. $N_{\text {atoms }}$ and $182 N_{\text {res }}$ represent the number of atoms and residues in the system, respectively. In equation (3), the $\mathrm{n}$ 183 is an integer defined as the magnitude of the threshold, which is a multiple of the $\alpha$.

184

$$
\begin{aligned}
& E_{\text {total }}=V_{\text {total_avg }}+n \times \alpha_{\text {totalral }} \\
& \alpha_{\text {totalral }}=0.2 \times N_{\text {atoms }}(\mathrm{N}=1,2,3 \ldots) \\
& E_{\text {dihedral }}=V_{\text {dihedral_avg }}+\left(3.5 \times N_{\text {res }}\right) \\
& \alpha_{\text {dihedral }}=3.5 \times \frac{N_{\text {res }}}{5}
\end{aligned}
$$

Herein, the equilibrated structures extracted from classical MD simulations were selected as the initial structures for the aMD simulations. The Dual-boost approach was applied by adding $\Delta V(r)$ to both the $E_{\text {total }}$ and $E_{\text {dihedral }}$ of the system. The $\Delta V(r)$ was obtained based on the $N_{\text {atoms }}$,

$N_{\text {res }}, V_{\text {total_avg }}$, and $V_{\text {dihedral_avg }}$ from the first $40 \mathrm{~ns}$ of the classical MD simulations. Then, 800 ns aMD simulations were employed using the dual-boost approach. During aMD simulations, the PME method was employed to estimate the long-range electrostatic interactions, with the cutoff parameter of nonbonded interaction set to $10 \AA$ (Essmann et al. 1995). SHAKE method was applied to constrain all covalent bonds connecting hydrogen atoms (Krautler et al. 2001). The Langevin temperature scalings was used to handle the temperature (Izaguirre et al. 2001). The coordinates for each complex were saved at an interval of $10 \mathrm{ps}$ and the trajectories were calculated using the CPPTRAJ module in Amber 18 program.

\section{Principal component analysis (PCA)}

PCA was performed on the trajectories from both classical MD and aMD simulations via the 
201 the translational and rotational motions of all protein $\mathrm{C}_{\alpha}$ atoms. Then, a covariance matrix $(3 \mathrm{~N} \times$

202

203

204

205

206

207

208

209

210

211

212

213

214

215

216

217

218

219

$$
\begin{aligned}
& \Delta G_{\text {bind }}=\Delta \mathrm{G}_{\mathrm{R}+\mathrm{L}}-\left(\Delta \mathrm{G}_{\mathrm{R}}+\Delta \mathrm{G}_{\mathrm{L}}\right)=\Delta \mathrm{E}_{\mathrm{MM}}+\Delta \mathrm{G}_{\text {sol }}-\mathrm{T} \Delta \mathrm{S} \\
& \Delta \mathrm{E}_{\mathrm{MM}}=\Delta \mathrm{E}_{\text {int }}+\Delta \mathrm{E}_{\mathrm{vdW}}+\Delta \mathrm{E}_{\text {elec }} \\
& \Delta \mathrm{G}_{\mathrm{sol}}=\Delta \mathrm{G}_{\mathrm{GB}}+\Delta \mathrm{G}_{\mathrm{SA}}
\end{aligned}
$$

\section{Binding free energy calculations based on classical MD simulations} the basis of the MM/GBSA method according to the following equations:

The MM/GBSA method is often used to perform classical MD simulations combined with freeenergy calculations, which can serve as an effective tool for quantitative prediction of proteinligand binding energies (Wang et al. 2019a). The binding free energies ( $\left.\Delta G_{\text {bind }}\right)$ are calculated on

In equation (8), $\Delta G_{\mathrm{R}+\mathrm{L}}, \Delta G_{\mathrm{R}}$, and $\Delta G_{\mathrm{L}}$ stand for the free energies of receptor-ligand complex, receptor and ligand, respectively. The sum of molecular mechanics interaction energy $\left(\Delta E_{\mathrm{MM}}\right)$, 
222

223

224

225

226

227

228

229

230

231

232

233

234

235

236

237

238

239

240

241

242

243

244

245

246

247

248

solvation energy $\left(\Delta G_{\mathrm{sol}}\right)$ and the change of the conformational entropy at temperature $\mathrm{T}(-T \Delta S)$ are equal to the sum of $\Delta G_{\mathrm{R}+\mathrm{L}}, \Delta G_{\mathrm{R}}$, and $\Delta G_{\mathrm{L}}$. In equation (9), $\Delta E_{\mathrm{MM}}$ is given as the sum of intermolecular interaction energy $\left(\Delta E_{\text {int }}\right)$, van der Waals energy $\left(\Delta E_{\mathrm{vdW}}\right)$, and electrostatic energy $\left(\Delta E_{\text {elec }}\right)$. The solvation free energy can be divided into polar $\left(\Delta G_{\mathrm{GB}}\right)$ and nonpolar $\left(\Delta G_{\mathrm{SA}}\right)$ and contributions (equation 10). Here, for each complex, 1,000 structures extracted from the classical MD simulations with a simulation time between 600 and 800 ns were utilized to conduct binding free energy calculations and per-residue energy decomposition. The $\Delta E_{\text {int }}$ was canceled as the single trajectory strategy was executed. Based on parameters by Onufriev et al., the $\Delta G_{\mathrm{GB}}$ was determined using a modified $\mathrm{GB}$ model $\left(\mathrm{GB}^{\mathrm{OBCl}}\right.$ ) (Onufriev et al. 2000). The $\Delta G_{\mathrm{SA}}$ was determined using the solvent accessible surface area (SASA) model $\left(\Delta G_{\mathrm{SA}}=\sigma^{*} \mathrm{SASA}\right)$. The parameter $\sigma$ was set to $0.0072 \mathrm{kcal} \cdot \mathrm{mol}^{-1} \cdot \AA^{-2}$. The $-T \Delta S$ was excluded from consideration because of relatively low prediction accuracy and high computational demand (Hou et al. 2011).

\section{Free energy landscape (FEL) calculation based on aMD}

The cumulant expansion to the second order method was employed to determine the FEL for each simulated complex from aMD simulations, because this method offers a good approximation for calculating the reweighting factor. In this study, the $\Delta V(r)$ combined with PC1 and PC2 from PCA of the aMD simulation trajectories were utilized to recover the FEL (Miao et al. 2014b; Roe \& Cheatham 2013).

Results

\section{Evaluation of the stability of simulated complexes from classical MD simulations}

To validate the docking results of the modeled complex of PARP-2 bound to Niraparib, structural alignments of the PARP-2/NMS-P118 and modeled PARP-2/Niraparib were performed with the corresponding crystal structures of PARP-1/NMS-P118 and PARP-1/Niraparib. As shown in Figure 2A, alignment of the crystal structures between PARP-1/NMS-P118 (PDB code: 5A00) and PARP-2/NMS-P118 (PDB code: 4ZZY) exhibited relatively high similarity, with a RMSD of 
249

250

251

252

253

254

255

256

257

258

259

260

261

262

263

264

265

266

267

268

269

270

\section{1}

272

273

274

275

$0.704 \AA$ for heavy atoms. Similarly, the alignment of the modeled complex of PARP-2/Niraparib with the crystal structure of PARP-1/Niraparib (PDB code: 4R6E) mostly showed similarities with only minor differences, with a RMSD of $0.767 \AA$ for heavy atoms (Figure 2B). These results suggest that the predicted model is sufficient to study the dynamic features through further MD simulations.

Firstly, $800 \mathrm{~ns}$ classical MD simulations for the modeled structure and the three crystal structures were performed. As a prerequisite for all further analyses, the dynamic stability of the simulated complexes was monitored by studying the RMSDs for all protein backbones $\left(\mathrm{C}_{\alpha}\right)$ atoms and all ligand heavy atoms for each complex with the starting structure as a function of simulation time. Theoretically, smaller fluctuations of RMSDs indicate greater stability of the complex. As shown in Figure 3, the time evolution of the RMSD values of $\mathrm{C}_{\alpha}$ atoms and ligand heavy atoms in each complex tend to converge after $\sim 100-300$ ns simulations. During the simulation of the last $400 \mathrm{~ns}$, the RMSD curves of both PARP-1 $\mathrm{C}_{\alpha}$ atoms displayed minor fluctuations $(<1 \AA)$, indicating that NMS-P118 constrained the protein structural flexibility of PARP-1. In contrast, those for both PARP-2 and NMS-P118 exhibited greater fluctuations compared to the complex of PARP-2/NMS-P118 (Figure 3B). This attested to the highly unstable nature of PARP-2 when it was bound to selective PARP-1 inhibitor NMS-P118. In comparison, the RMSD curves for Niraparib in both PARP-1 and PARP-2 oscillated with minute fluctuations $(<1 \AA)$ during the last $400 \mathrm{~ns}$ simulation, indicating that the non-selective drug Niraparib constrained the structural flexibility of both PARP-1 and PARP-2. Based on these results, the structural and energetic properties for each complex were further analyzed in detail.

\section{Dynamic features of each complex from classical MD simulations}

The different flexibility for NMS-P118 and Niraparib in different proteins may result in different protein conformational transitions. To characterize the protein conformational transitions over time, PCA was performed to identify the trend in large-scale collective motions. Generally, the eigenvectors with the highest eigenvalues capture the majority of variance in the original protein conformational 
276

277

278

279

280

281

282

283

284

285

286

287

288

289

290

291

292

293

294

295

296

297

distributions (Mashiko 2018; Sittel et al. 2017). Herein, the protein conformational ensembles were investigated by projecting the first two principal components (PC1 and PC2) from classical MD simulations onto a two-dimensional space. Generally, when PC1 and PC2 are plotted against each other, structures with high similarity are clustered together. As a result, a cluster represents a different state of protein conformation. As shown in Figure 4, the protein conformational distributions for each complex were dynamic during $800 \mathrm{~ns}$ classical MD simulations and eventually reached overall stability. It is apparent that the conformational distributions of PARP1/NMS-P118 were remarkably different from those of PARP-2/NMS-P118, while those for PARP1/Niraparib and PARP-2/Niraparib were also different. Analysis of the protein conformational distributions clearly showed that PARP-2/NMS-P118 samples a wider conformational space compared to PARP-1/NMS-P118. However, those for PARP-1/Niraparib and PARP-2/Niraparib shared a certain degree of similarity. These results demonstrate that the selective PARP-1 inhibitor NMS-P118 bound to PARP-1 had a different protein conformational flexibility compared to PARP-1, not the non-selective drug Niraparib.

To further investigate the correlation between the motion of the residues in the proteins, DCC analysis was performed. As plotted in Figure 5, the direction of correlation is represented by a color gradient ranging from blue (negative correlation) to red (positive correlation). The correlation coefficient $(-1$ to +1$)$, corresponding to three different colors: dark blue $(-0.25$ to -1$)$ represents anti-correlation; dark red (0.25 to 1$)$ represents positive correlation; blue represents anticorrelation (-0.25 to -1$)$; and light red or light blue $(-0.25$ to +0.25$)$ represents weak or nocorrelation. It can be observed that both the red and blue regions in PARP-2/NMS-P118 were larger and more intense than those in PARP-1/NMS-P118, implying elevated correlation or anticorrelation motions in PARP-2/NMS-P118 (Figure 5A and 5B). In comparison, color regions for PARP-1 and PARP-2 bound to the non-selective drug Niraparib were quite similar (Figure 5C and 5D). These results indicate that the relative motions of different protein subdomains may correlate with different protein flexibility, which was responsible for drug selectivity.

To further highlight the key sub-domains, the $\mathrm{C}_{\alpha}$ of RMSF analyses were conducted. Low 
303

304

305

306

307

308

309

310

311

312

313

314

315

316

317

318

319

320

321

322

323

324

325

326

327

328

329

RMSF values of residues represented less flexibility, whereas high RMSF values indicated greater fluctuations in relation to their average position during simulation. As shown in Figure 6, the RMSFs of PARP-1/NMS-P118 showed a high degree of similarity with those of PARP1/Niraparib. However, the helix $\alpha$ F connected with loop 347-356 in PARP-2 exhibited amplified fluctuations when it binds to the selective PARP-1 inhibitor NMS-P118 compared with the nonselective drug Niraparib. The above results indicate that the binding of selective PARP-1 inhibitor NMS-P118 leads to increased fluctuations of PARP-2 (especially the $\alpha \mathrm{F}$ and loop 347-356), which might be the dominating driving force for the redistributions of free energies.

\section{Binding free energy calculations using the MM/GBSA method based on classical MD} simulation trajectories

MM/GBSA binding free energy calculations based on classical MD simulation trajectories were applied for the assessment of the energy properties of NMS-P118 and Niraparib when they were bound with PARP-1 or PARP-2. As listed in Table 1, the predicted binding free energies $\left(\Delta G_{\text {binding }}\right)$ for PARP-1/NMS-P118, PARP-2/NMS-P118, PARP-1/Niraparib and PARP-2/Niraparib were $46.06 \pm 0.15,-36.40 \pm 0.14,-44.35 \pm 0.22$, and $-43.89 \pm 0.24 \mathrm{kcal} / \mathrm{mol}$, respectively. It is clear that the $\Delta G_{\text {binding }}$ was highly correlated with the experimental data reported, and that for each system,

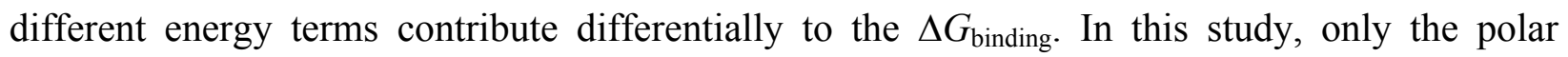
contributions $\left(\Delta E_{\text {elec }}+\Delta G_{\mathrm{GB}}\right)$ for the most vital factor were discussed. The polar contributions for the PARP-1/NMS-P118 and PARP-2/NMS-P118 were significantly different at $10.65 \pm 0.34$ and $19.29 \pm 0.16 \mathrm{kcal} / \mathrm{mol}$, respectively. In comparison, those for PARP-1/Niraparib and PARP2/Niraparib were quite similar at $9.72 \pm 0.29$ and $9.84 \pm 0.17 \mathrm{kcal} / \mathrm{mol}$. The non-polar contributions $\left(\Delta E_{\mathrm{vdW}}+\Delta G_{\mathrm{SA}}\right)$ for PARP-1/NMS-P118 and PARP-2/NMS-P118 (-56.70 \pm 0.12 and $-55.69 \pm 0.19$ $\mathrm{kcal} / \mathrm{mol}$ ) were almost identical with those for PARP-1/Niraparib and PARP-2/Niraparib ($53.92 \pm 0.26$ and $-53.72 \pm 0.41 \mathrm{kcal} / \mathrm{mol})$. Taken together, these results demonstrate that the polar contribution has a significant impact on drug selectivity to PARP-1 and PARP-2.

To gain further insights into the vital residues in drug selectivity, per-residue decomposition 
330 based on the MM/GBSA method was employed to assess residue contributions to the binding of 331 the protein-ligand complexes. Per-residue energy differences between Niraparib and NMS-P118

332

333

334

335

336

337

338

339

340

341

342

343

344

345

346

347 systems $\left(\Delta \Delta G=\Delta G_{\text {Niraparib }}-\Delta G_{\text {NMS-P118 }}\right)$ were plotted to identify the key residues. Negative values represent the residues of Niraparib formed stronger interactions with the protein than those of NMS-P118, whereas positive values indicated quite the opposite, namely, that the residues of Niraparib formed weaker interactions with the protein than those of NMS-P118. As shown in Figure 7A, the differences of $\Delta \Delta G$ between PARP-1/NMS-P118 and PARP-1/Niraparib were quite small with $\Delta \Delta G$ less than $0.5 \mathrm{kcal} / \mathrm{mol}$. Alignment of the representative structures of PARP1/NMS-P118 and PARP-1/Niraparib were highly similar (Figure 7B). However, the residues of Ser-328, Gln-322, Glu-335, and Tyr-455 formed significantly stronger interactions with Niraparib than with NMS-P118 in PARP-2 (Figure 7C). Notably, the key residues of Ser-328, Gln-322 and Glu-335 were located in the helix $\alpha \mathrm{F}$, which exhibited amplified fluctuations in RMSF analysis. This may be due to the fact that the distinctive flexibility of the helix $\alpha \mathrm{F}$ in PARP-2 bound to selective PARP-1 inhibitors induced energetic redistributions.

\section{Evaluation of the stability of the simulated complexes from aMD simulations}

To explore the conformational behaviors in greater detail, aMD simulations were first employed. Thereafter, the RMSDs of $\mathrm{C}_{\alpha}$ atoms and ligand heavy atoms were monitored. As shown in Figure 8 , the RMSD values of $\mathrm{C}_{\alpha}$ atoms and ligand heavy atoms for each complex reached equilibrium after 120-200 ns aMD simulations, suggesting that simulated complexes became dynamically stable through $800 \mathrm{~ns}$ aMD simulations. Interestingly, the fluctuations of selective PARP-1 inhibitor NMS-P118 bound to PARP-1 were much smaller than when bound to PARP-2. In contrast, the fluctuations of non-selective drug Niraparib were similar in both PARP-1 and PARP2. A comparison of these results revealed that the selective PARP-1 inhibitor allowed for larger protein conformational changes of PARP-2. These findings were corroborated by the DCC analysis, which further revealed that the red and blue regions in PARP-2/NMS-P118 were both larger and more intense than those in PARP-1/NMS-P118, while those for PARP-1 and PARP-2 
357

358

359

360

361

362

363

364

365

366

367

368

369

370

371

372

373

374

375

376

377

378

379

380

381

382

383

bound to Niraparib were quite similar (Figure 9).

Based on the above findings, PCA was used to identify the various protein conformations obtained during the aMD simulations. As shown in Figure 10, the protein conformations of for each complex was characterized by dynamic fluctuations during $800 \mathrm{~ns}$ aMD simulations. Similar with the results from classical MD simulaitons, the conformational distributions of PARP-1/NMSP118 were remarkably different from those of PARP-2/NMS-P118 (Figure 10A-B). Meanwhile, those for PARP-1/Niraparib and PARP-2/Niraparib were also different (Figure 10C-D). Compared to the PARP-1/NMS-P118 complex, the PARP-2/NMS-P118 complex exhibited more structural clusters and a wider range of conformational distributions (Figure 10A-B). However, the conformational distributions for Niraparib bound to PARP-1 and PARP-2 had a somewhat similar range (Figure 10C-D), suggesting that the selective PARP-1 inhibitors could stabilize the protein conformation of PARP-1. These results were in agreement with the RMSDs, DCC and PCA analyses from the classical MD simulations.

The FEL was employed to further demonstrate the relationship between the changes of conformation and energy (Figure 11). Generally, more energy wells (dark blue regions) represent greater conformational changes of the protein during aMD simulation (Han et al. 2019; Miao et al. 2014a). As shown in Figure 11A, only a deep energy well for NMS-P118 bound to PARP-1 was observed throughout the whole 800 ns aMD simulation. In contrast, two major deep energy wells with a much wider range of distributions were observed for the complex of PARP-1/NMS-P118 (Figure 11B). Nevertheless, those for Niraparib bound to PARP-1 and PARP-2 showed a similar range of distributions and both were confined to a single deep energy. These results highlight the unstable nature of PARP-2 bound to selective PARP-1 inhibitor NMS-P118.

\section{Discussion}

PARP-1 inhibitors have been widely studied as potential cancer therapeutics for breast and ovarian cancers (Min \& Im 2020). The reported clinical candidates and preclinical PARP-1 inhibitors were designed with the purpose of imitating the nicotinamide portion of nicotinamide adenine 
dinucleotide $\left(\mathrm{NAD}^{+}\right)$, with which they compete for the corresponding PARP-1 binding site. A number of recent studies have indicated that due to their high sequence and structural similarity (Figure 1A-C), non-selective PARPs drugs may result in potentially undesirable side effects, especially between PARP-1 and PARP-2 (Eltze et al. 2008; Fatima et al. 2014; Papeo et al. 2015). In this aspect, the ideal drug candidate should be a highly selective PARP-1 inhibitor with greater subtype specificity. Until now, only a few highly selective PARP-1 inhibitors have been reported. However, even fewer computational modeling researches have been conducted to elucidate their underlying selective mechanisms.

In the present study, a comprehensive molecular computational method was employed to demonstrate the selective mechanisms via two representative inhibitors (NMS-P118, Niraparib) with different selectivity to PARP-1 and PARP-2 (Figure 1D-E). Initially, molecular docking was applied to predict the complex of PARP-2/Niraparib. To examine the prediction accuracy, the predicted and the crystal structures were aligned. The RMSDs between the predict pose (PARP2/Niraparib) and the crystal structure (PARP-1/Niraparib) were highly similar, a finding that was consistent with the results observed by alignment of crystal structures of NMS-P118 bound to PARP-1 and PARP-2 (Figure 2). Based on the above findings, these structures were employed to further explore the dynamic behavior via classical MD simulations and aMD simulations. Classical MD simulations in conjunction with the RMSD, PCA, and DCC analyses provided compelling evidence that the conformation fluctuations were different for PARP-2 bound to selective PARP1 inhibitors and PARP-2 bound to non-selective inhibitor (Figure 3-5). Further RMSF analysis revealed that the major structural variations were the conformational changes of the helix $\alpha \mathrm{F}$, which may account for drug selectivity (Figure 6). According to the binding free energy calculations, the polar contributions had an obvious impact on drug selectivity (Table 1). Perresidue decomposition analysis further revealed that drug selectivity was primarily controlled by the residues of Ser-328, Gln-322, Glu-335, and Tyr-455, most of which are located in the helix $\alpha \mathrm{F}$ (Figure 7).

As there are possible energy barriers between various meta-stable states, the classical MD 
411 simulations may still not be sufficient to sample the possible conformations (Hamelberg et al.

412 2004; Miao et al. 2014a). Therefore, an enhanced sampling technique that can sample the protein

413 conformation at various meta-stable states is still required. Most of enhanced sampling techniques

414 often require expert knowledge of the studied complexes, as these techniques require reaction

415 coordinates. This limitation is overcome by the aMD simulations, which can explore the

416 conformational behavior of the biomacromolecule without this requirement (Hamelberg et al.

417 2004; Miao et al. 2014a). Therefore, within the framework of this study, aMD simulation was

418 employed to further sample the possible conformational ensembles. The results of RMSD, PCA,

419 DCC analyses from aMD simulations indicated that the selective PAPR-1 inhibitor NMS-P118

420 may significantly disrupt the stability of the PARP-2 protein, not the non-selective drug Niraparib,

421 a finding that was consistent with the results from classical MD simulations (Figure 8-10). The

422 FEL analysis further demonstrated the unstable nature of PAPR-2 bound to the selective PAPR-1

423 inhibitors NMS-P118 (Figure 11). The preferential binding of NMS-P118 to PARP-1 takes

424 precedence over PARP-2, resulting in a better inhibitory effect of PARP-1 than PARP-2, and

425 greater PARP-1 selectivity. In summary, more selective PAPR-1 inhibitors may be required to

426 evaluate the above findings via molecular modeling, which might help facilitate the rational design

427 of high selective PAPR-1 inhibitors.

428

429 Conclusions

430 Multiple computational techniques were employed to conduct an exploration of the selective 431 mechanisms of inhibitors underlying PARP-1 and PARP-2. The results from classical MD 432 simulations offered compelling evidence that preferential NMS-P118 binding to PARP-1 over 433 PARP-2 was controlled by the protein conformational changes of helix $\alpha \mathrm{F}$, which lead to decreased 434 polar contributions. These findings were further corroborated by the RMSDs, DCC, PCA analyses 435 from aMD simulations. FEL results from aMD simulations further suggested that PAPR-2 bound 436 to the selective PARP-1 inhibitor NMS-P118, but not the non-selective drug Niraparib, underwent 437 large conformational changes. Taken together, these results may prove conducive to the design of 
438

439

440

441

442

443

444

445

446

447

448

449

450

451

452

453

454

455

456

457

458

459

460

461

462

463

464

465

466

467

468

469

470

471

472

473

more selective PAPR-1 inhibitors with fewer potential side effects.

\section{Acknowledgments}

This work was supported by the Natural Science Foundation of Zhejiang Province of China (LY20H160011).

\section{Conflicts of Interest}

The authors declare that they have no competing interests.

\section{References}

Begg CB, Haile RW, Borg A, Malone KE, Concannon P, Thomas DC, Langholz B, Bernstein L, Olsen JH, Lynch CF, Anton-Culver H, Capanu M, Liang X, Hummer AJ, Sima C, and Bernstein JL. 2008. Variation of breast cancer risk among BRCA1/2 carriers. JAMA 299:194-201. 10.1001/jama.2007.55-a

Dolinsky TJ, Nielsen JE, McCammon JA, and Baker NA. 2004. PDB2PQR: an automated pipeline for the setup of Poisson-Boltzmann electrostatics calculations. Nucleic Acids Res 32:W665-667. 10.1093/nar/gkh381

Eltze T, Boer R, Wagner T, Weinbrenner S, McDonald MC, Thiemermann C, Burkle A, and Klein T. 2008. Imidazoquinolinone, imidazopyridine, and isoquinolindione derivatives as novel and potent inhibitors of the poly(ADP-ribose) polymerase (PARP): a comparison with standard PARP inhibitors. Mol Pharmacol 74:1587-1598. 10.1124/mol.108.048751

Essmann U, Perera L, Berkowitz ML, Darden T, Lee H, and Pedersen LG. 1995. A smooth particle mesh Ewald method. The Journal of chemical physics 103:8577-8593.

Farres J, Martin-Caballero J, Martinez C, Lozano JJ, Llacuna L, Ampurdanes C, Ruiz-Herguido C, Dantzer F, Schreiber V, Villunger A, Bigas A, and Yelamos J. 2013. Parp-2 is required to maintain hematopoiesis following sublethal gamma-irradiation in mice. Blood 122:44-54. 10.1182/blood-2012-12-472845

Fatima S, Jatavath MB, Bathini R, Sivan SK, and Manga V. 2014. Multiple receptor conformation docking, dock pose clustering and 3D QSAR studies on human poly(ADP-ribose) polymerase-1 (PARP-1) inhibitors. J Recept Signal Transduct Res 34:417-430. 10.3109/10799893.2014.917323

Gunderson CC, and Moore KN. 2015. Olaparib: an oral PARP-1 and PARP-2 inhibitor with promising activity in ovarian cancer. Future Oncol 11:747-757. 10.2217/fon.14.313

Hamelberg D, Mongan J, and McCammon JAJTJocp. 2004. Accelerated molecular dynamics: a promising and efficient simulation method for biomolecules. 120:11919-11929.

Han Y, Wu Y, Xu Y, Guo W, Zhang N, and Wang X. 2019. Molecular mechanism of point mutation-induced Monopolar spindle 1 (Mps1/TTK) inhibitor resistance revealed by a comprehensive molecular modeling study. PeerJ 7:e6299. 10.7717/peerj.6299

Hou T, Wang J, Li Y, and Wang W. 2011. Assessing the performance of the MM/PBSA and MM/GBSA methods. 1. The accuracy of binding free energy calculations based on molecular dynamics simulations. 
J Chem Inf Model 51:69-82. 10.1021/ci100275a

Ison G, Howie LJ, Amiri-Kordestani L, Zhang L, Tang S, Sridhara R, Pierre V, Charlab R, Ramamoorthy A, Song P, Li F, Yu J, Manheng W, Palmby TR, Ghosh S, Horne HN, Lee EY, Philip R, Dave K, Chen XH, Kelly SL, Janoria KG, Banerjee A, Eradiri O, Dinin J, Goldberg KB, Pierce WF, Ibrahim A, Kluetz PG, Blumenthal GM, Beaver JA, and Pazdur R. 2018. FDA Approval Summary: Niraparib for the Maintenance Treatment of Patients with Recurrent Ovarian Cancer in Response to Platinum-Based Chemotherapy. Clin Cancer Res 24:4066-4071. 10.1158/1078-0432.CCR-18-0042

Izaguirre JA, Catarello DP, Wozniak JM, and Skeel RD. 2001. Langevin stabilization of molecular dynamics. The Journal of chemical physics 114:2090-2098.

Jubin T, Kadam A, Jariwala M, Bhatt S, Sutariya S, Gani AR, Gautam S, and Begum R. 2016. The PARP family: insights into functional aspects of poly (ADP-ribose) polymerase-1 in cell growth and survival. Cell Prolif 49:421-437. 10.1111/cpr.12268

Kim DS, Challa S, Jones A, and Kraus WL. 2020. PARPs and ADP-ribosylation in RNA biology: from RNA expression and processing to protein translation and proteostasis. Genes Dev 34:302-320. 10.1101/gad.334433.119

Krautler V, Van Gunsteren WF, and Hunenberger PH. 2001. A fast SHAKE: Algorithm to solve distance constraint equations for small molecules in molecular dynamics simulations. Journal of Computational Chemistry 22:501-508. Doi 10.1002/1096-987x(20010415)22:5<501::Aid-Jcc1021>3.0.Co;2-V

Lin J, Yang X, and Zhao H. 2019. BRCA Mutations and Homologous Recombination Repair Deficiency in Treatment With Niraparib Combined With Pembrolizumab. JAMA Oncol. 10.1001/jamaoncol.2019.4595

Lin KY, and Kraus WL. 2017. PARP Inhibitors for Cancer Therapy. Cell 169:183. 10.1016/j.cell.2017.03.034

Maier JA, Martinez C, Kasavajhala K, Wickstrom L, Hauser KE, Simmerling CJJoct, and computation. 2015. ff14SB: improving the accuracy of protein side chain and backbone parameters from ff99SB. 11:36963713.

Mashiko TJJoCC, Japan. 2018. Principal Component Analysis for Molecular Dynamics Simulation of the Crystal Phase Transition. 17:196-198.

McCann KE. 2019. Advances in the use of PARP inhibitors for BRCA1/2-associated breast cancer: talazoparib. Future Oncol 15:1707-1715. 10.2217/fon-2018-0751

Miao Y, Nichols SE, and McCammon JA. 2014a. Free energy landscape of G-protein coupled receptors, explored by accelerated molecular dynamics. Phys Chem Chem Phys 16:6398-6406. $10.1039 / \mathrm{c} 3 \mathrm{cp} 53962 \mathrm{~h}$

Miao Y, Sinko W, Pierce L, Bucher D, Walker RC, and McCammon JA. 2014b. Improved Reweighting of Accelerated Molecular Dynamics Simulations for Free Energy Calculation. $J$ Chem Theory Comput 10:2677-2689. 10.1021/ct500090q

Min A, and Im SA. 2020. PARP Inhibitors as Therapeutics: Beyond Modulation of PARylation. Cancers (Basel) 12. $10.3390 /$ cancers 12020394

Morris GM, Huey R, Lindstrom W, Sanner MF, Belew RK, Goodsell DS, and Olson AJ. 2009. AutoDock4 and AutoDockTools4: Automated docking with selective receptor flexibility. Journal of computational chemistry 30:2785-2791. 10.1002/jcc.21256

Navarro J, Gozalbo-Lopez B, Mendez AC, Dantzer F, Schreiber V, Martinez C, Arana DM, Farres J, Revilla- 
Nuin B, Bueno MF, Ampurdanes C, Galindo-Campos MA, Knobel PA, Segura-Bayona S, MartinCaballero J, Stracker TH, Aparicio P, Del Val M, and Yelamos J. 2017. PARP-1/PARP-2 double deficiency in mouse $\mathrm{T}$ cells results in faulty immune responses and T lymphomas. Sci Rep 7:41962. $10.1038 /$ srep41962

Onufriev A, Bashford D, and David A. 2000. Modification of the generalized Born model suitable for macromolecules. $J$ Phys Chem 104:3712-3720.

Papeo G, Posteri H, Borghi D, Busel AA, Caprera F, Casale E, Ciomei M, Cirla A, Corti E, D'Anello M, Fasolini M, Forte B, Galvani A, Isacchi A, Khvat A, Krasavin MY, Lupi R, Orsini P, Perego R, Pesenti E, Pezzetta D, Rainoldi S, Riccardi-Sirtori F, Scolaro A, Sola F, Zuccotto F, Felder ER, Donati D, and Montagnoli A. 2015. Discovery of 2-[1-(4,4-Difluorocyclohexyl)piperidin-4-yl]-6-fluoro-3-oxo-2,3dihydro-1H-isoind ole-4-carboxamide (NMS-P118): A Potent, Orally Available, and Highly Selective PARP-1 Inhibitor for Cancer Therapy. J Med Chem 58:6875-6898. 10.1021/acs.jmedchem.5b00680

Pettersen EF, Goddard TD, Huang CC, Couch GS, Greenblatt DM, Meng EC, and Ferrin TE. 2004. UCSF Chimera--a visualization system for exploratory research and analysis. J Comput Chem 25:1605-1612. 10.1002/jcc. 20084

Pfeffer CM, Ho BN, and Singh ATK. 2017. The Evolution, Functions and Applications of the Breast Cancer Genes BRCA1 and BRCA2. Cancer Genomics Proteomics 14:293-298. 10.21873/cgp.20040

Praprotnik M, Delle Site L, and Kremer KJTJocp. 2005. Adaptive resolution molecular-dynamics simulation: Changing the degrees of freedom on the fly. 123:224106.

Roe DR, and Cheatham TE, 3rd. 2013. PTRAJ and CPPTRAJ: software for processing and analysis of molecular dynamics trajectory data. $J$ Chem Theory Comput 9:3084-3095. 10.1021/ct400341p

Siegel RL, Miller KD, and Jemal A. 2018. Cancer statistics, 2018. CA Cancer J Clin 68:7-30. $10.3322 /$ caac. 21442

Sittel F, Filk T, and Stock GJTJocp. 2017. Principal component analysis on a torus: Theory and application to protein dynamics. $147: 244101$.

Skjaerven L, Yao XQ, Scarabelli G, and Grant BJ. 2014. Integrating protein structural dynamics and evolutionary analysis with Bio3D. BMC Bioinformatics 15:399. 10.1186/s12859-014-0399-6

Thorsell AG, Ekblad T, Karlberg T, Low M, Pinto AF, Tresaugues L, Moche M, Cohen MS, and Schuler H. 2017. Structural Basis for Potency and Promiscuity in Poly(ADP-ribose) Polymerase (PARP) and Tankyrase Inhibitors. J Med Chem 60:1262-1271. 10.1021/acs.jmedchem.6b00990

Vassetti D, Pagliai M, Procacci PJJoct, and computation. 2019. Assessment of GAFF2 and OPLS-AA general force fields in combination with the water models TIP3P, SPCE, and OPC3 for the solvation free energy of druglike organic molecules. 15:1983-1995.

Wang E, Sun H, Wang J, Wang Z, Liu H, Zhang JZH, and Hou T. 2019a. End-Point Binding Free Energy Calculation with MM/PBSA and MM/GBSA: Strategies and Applications in Drug Design. Chem Rev 119:9478-9508. 10.1021/acs.chemrev.9b00055

Wang J, Cieplak P, and Kollman PAJJocc. 2000. How well does a restrained electrostatic potential (RESP) model perform in calculating conformational energies of organic and biological molecules? 21:10491074.

Wang Y, Luo W, and Wang Y. 2019b. PARP-1 and its associated nucleases in DNA damage response. DNA Repair (Amst) 81:102651. 10.1016/j.dnarep.2019.102651 
556 Yelamos J, Farres J, Llacuna L, Ampurdanes C, and Martin-Caballero J. 2011. PARP-1 and PARP-2: New 557 players in tumour development. Am J Cancer Res 1:328-346.

558 


\section{Table 1 (on next page)}

Binding free energies of NMS-P118 and Niraparib in PARP-1 and PARP-2 $(\mathrm{kcal} / \mathrm{mol})$ 
1 Table 1. Binding free energies of NMS-P118 and Niraparib in PARP-1 and PARP-2 (kcal/mol)

\begin{tabular}{|c|c|c|c|c|}
\hline Ligand & \multicolumn{2}{|c|}{ NMS-P118 } & \multicolumn{2}{|c|}{ Niraparib } \\
\hline Protein & PARP-1 & PARP-2 & PARP-1 & PARP-2 \\
\hline$\Delta E_{\mathrm{vdW}}$ & $-50.94 \pm 0.14$ & $-49.87 \pm 0.12$ & $-48.65 \pm 0.16$ & $-48.46 \pm 0.14$ \\
\hline$\Delta E_{\mathrm{elec}}$ & $-85.06 \pm 0.70$ & $-54.88 \pm 0.53$ & $-116.24 \pm 0.91$ & $-86.98 \pm 1.05$ \\
\hline$\Delta G_{\mathrm{GB}}$ & $95.71 \pm 0.68$ & $74.17 \pm 0.52$ & $125.96 \pm 0.78$ & $96.82 \pm 0.89$ \\
\hline$\Delta G_{\mathrm{SA}}$ & $-5.76 \pm 0.01$ & $-5.82 \pm 0.01$ & $-5.27 \pm 0.01$ & $-5.26 \pm 0.01$ \\
\hline$\Delta E_{\text {non-polar }}$ & $-56.70 \pm 0.12$ & $-55.69 \pm 0.19$ & $-53.92 \pm 0.26$ & $-53.72 \pm 0.41$ \\
\hline$\Delta E_{\text {polar }}$ & $10.65 \pm 0.34$ & $19.29 \pm 0.16$ & $9.72 \pm 0.29$ & $9.84 \pm 0.17$ \\
\hline$\Delta G_{\text {bind }}$ & $-46.06 \pm 0.15$ & $-36.40 \pm 0.14$ & $-44.35 \pm 0.22$ & $-43.89 \pm 0.24$ \\
\hline
\end{tabular}

$2 \Delta E_{\mathrm{vdw}}$ : Van der Waals energy; $\Delta E_{\text {ele: }}$ Electrostatic energy; $\Delta G_{\mathrm{GB}}$ : Electrostatic contribution to solvation;

$3 \Delta G_{\mathrm{SA}}$ : Non-polar contribution to solvation; $\Delta E_{\text {non-polar: }}$ Non-polar interaction; $\Delta E_{\text {polar }}$ : polar interaction; $\Delta G_{\text {bind: }}$

4 Binding free energy;

5 
Figure 1

Alignment of the crystal structures of PARP-1 and PARP-2, and representative inhibitors.

(A) Overview of the crystal structures of PARP-1 (magenta, PDB code: 5A00) and PARP-2 (green, PDB code: 4ZZY), the binding pocket is colored blue. (B) Close up view of the active site of PARP-1, the residues of Glu-988 is vital for catalysis. (C) Close up view of the active site of PARP-2, the residues of Glu-558 is vital for catalysis. (D) Chemical structure of NMSP118. (E) Chemical structure of Niraparib. 
A

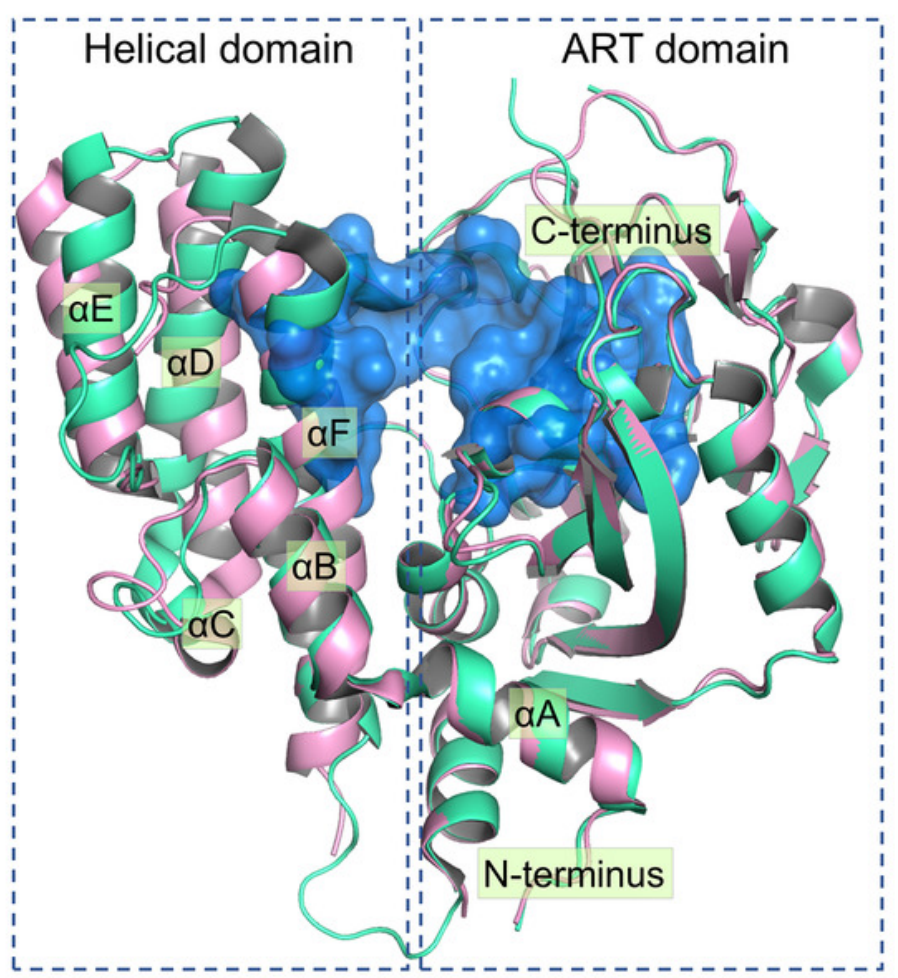

D

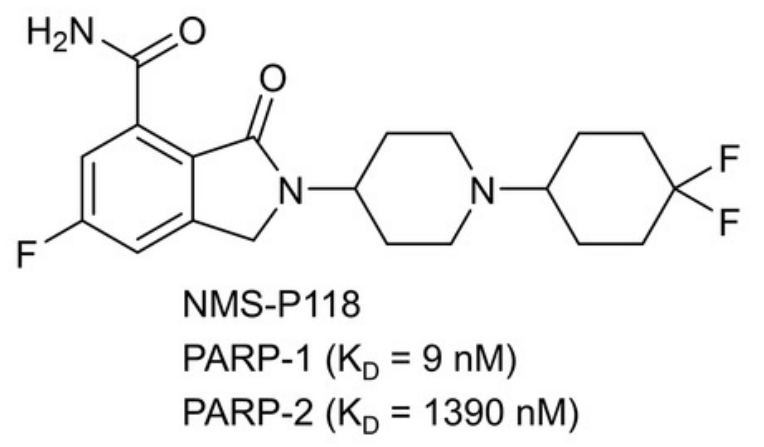

B

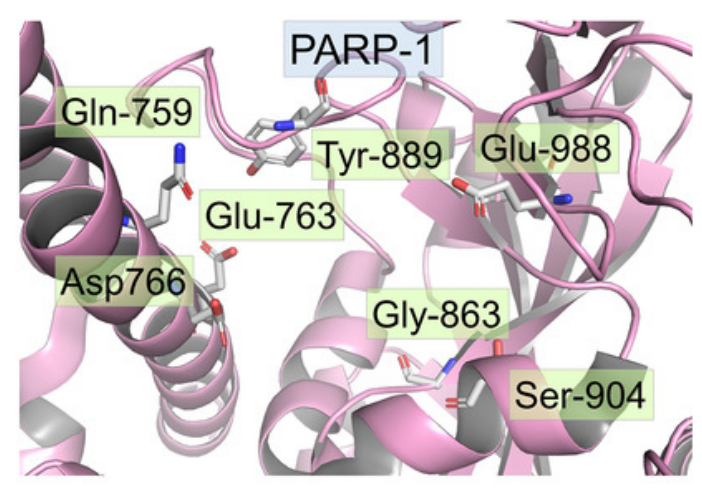

C

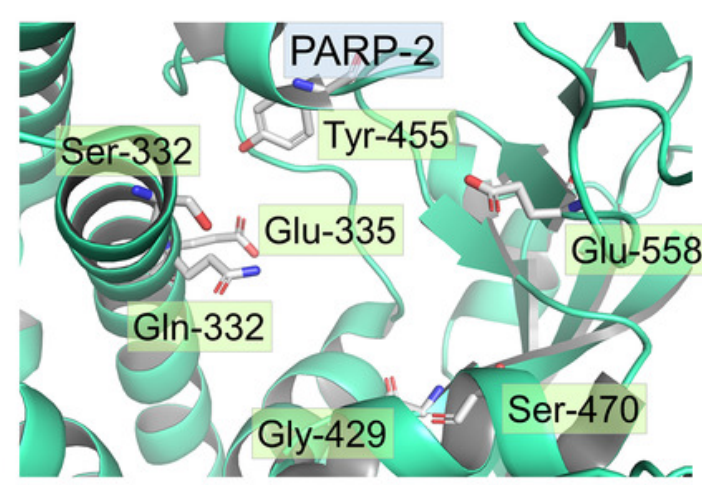

$E$

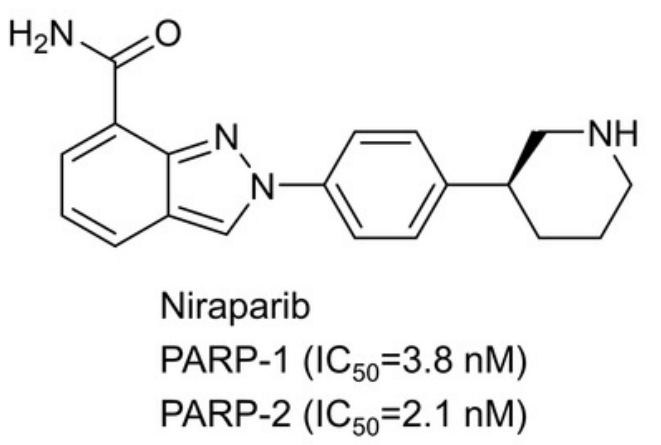


Figure 2

Validation of modeling results.

(A) Alignment of the crystal structures of PARP-1/NMS-P118 and PARP-2/NMS-P118.

Alignment of the crystal structure of PARP-1/Niraparib and the modeled structure of PARP-2/Niraparib.

A

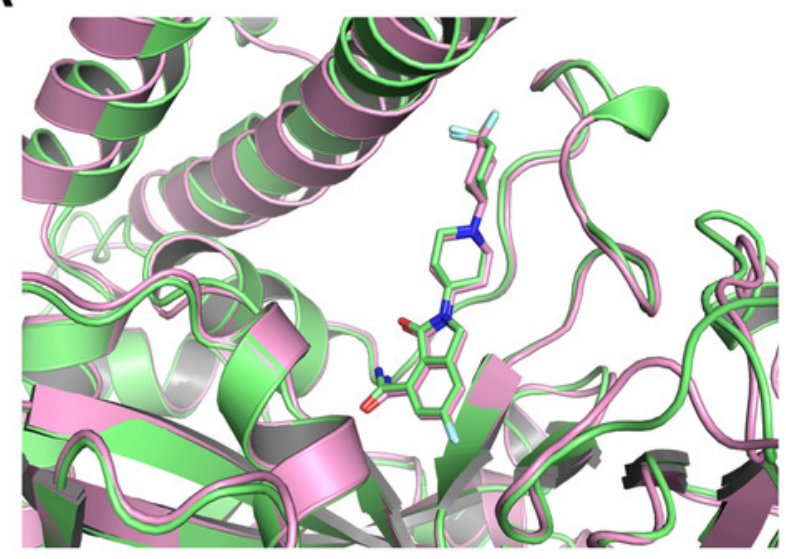

PARP-1/NMS-P118 (magenta) PARP-2/ NMS-P118 (green)
$\mathrm{B}$

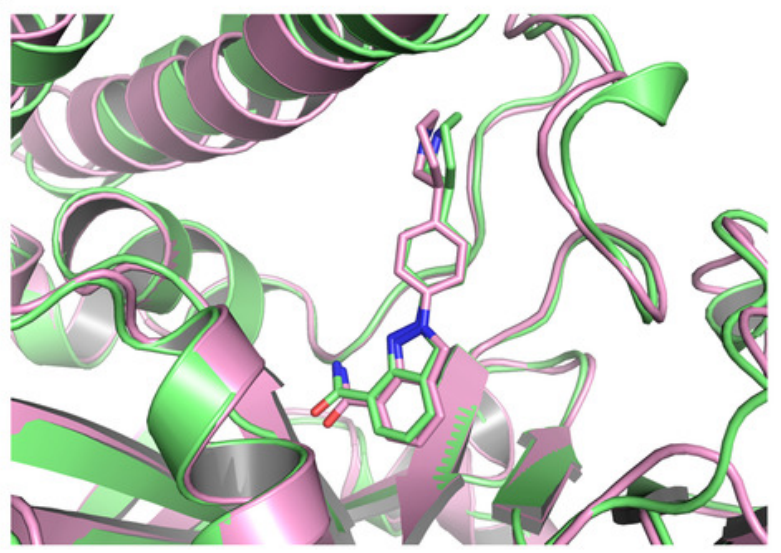

PARP-1/Niraparib (magenta) PARP-2/Niraparib (green) 
Figure 3

RMSD values of the protein backbones atoms and ligand heavy atoms from the $800 \mathrm{~ns}$ classical MD simulations.

(A) PARP-1/NMS-P118. (B) PARP-2/NMS-P118. (C) PARP-1/Niraparib. (D) PARP-2/Niraparib.

A

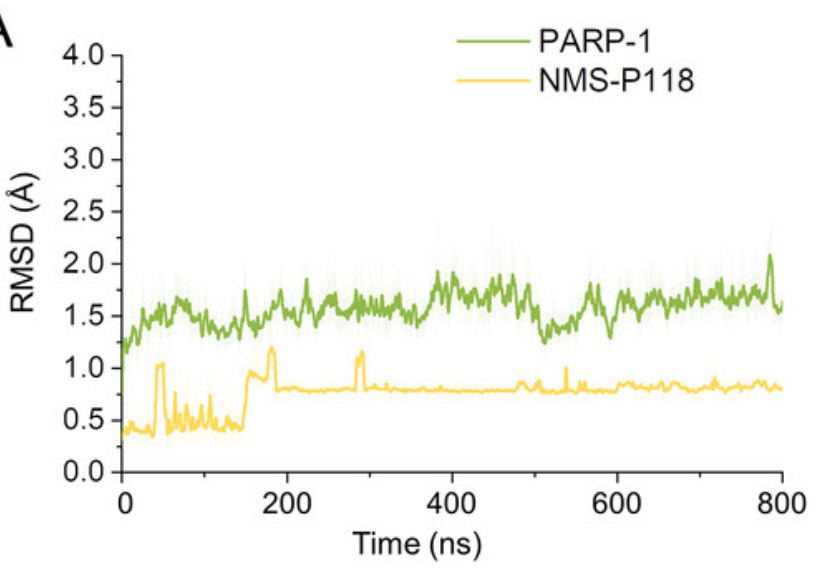

C

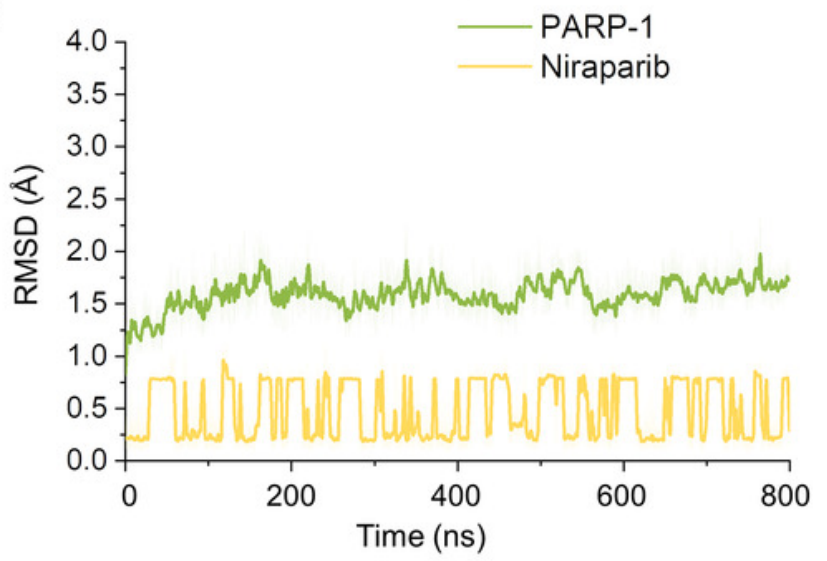

B
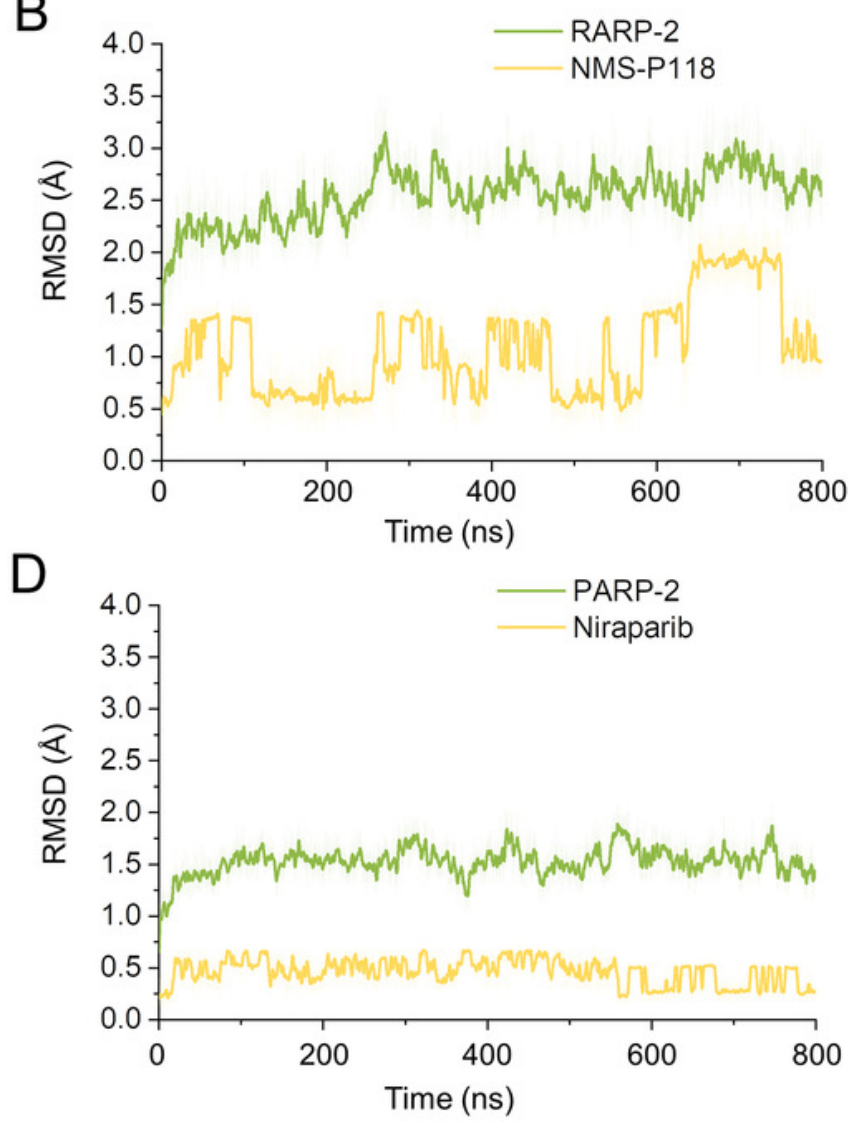
Figure 4

The top two ranked principal components (PC1, PC2) are plotted against each other from the 800 ns classical MD simulations.

(A) PARP-1/NMS-P118. (B) PARP-2/NMS-P118. (C) PARP-1/Niraparib. (D) PARP-2/Niraparib.

A

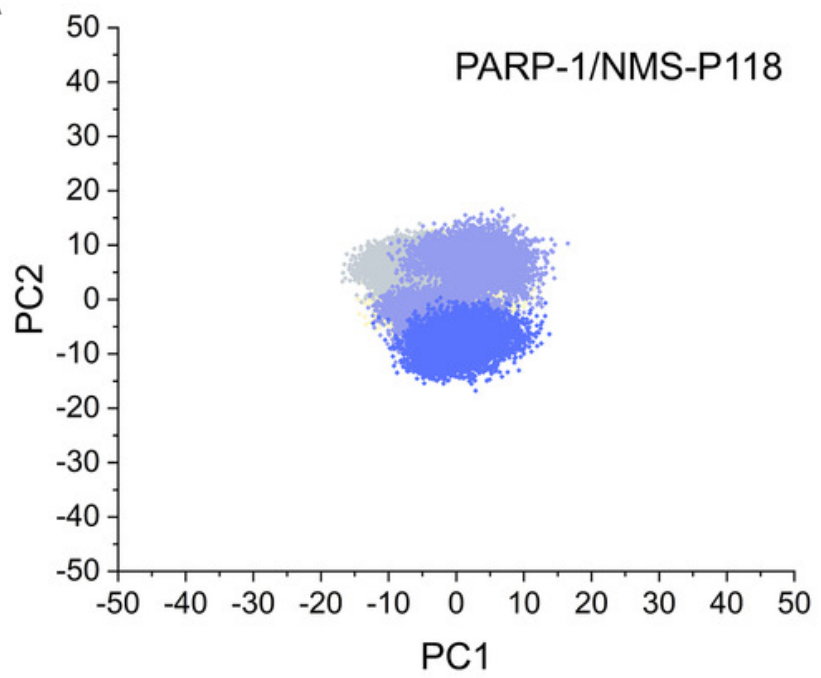

C

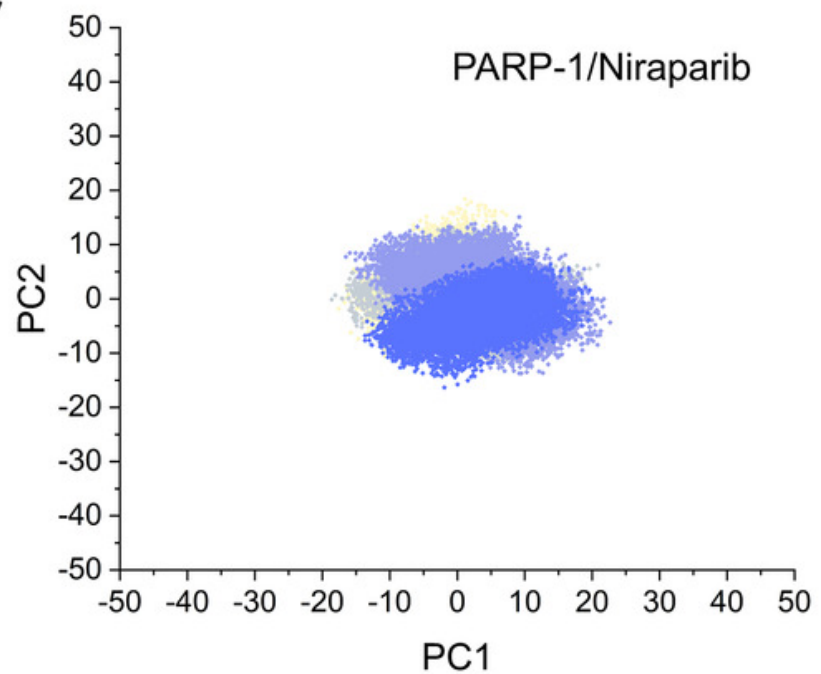

B

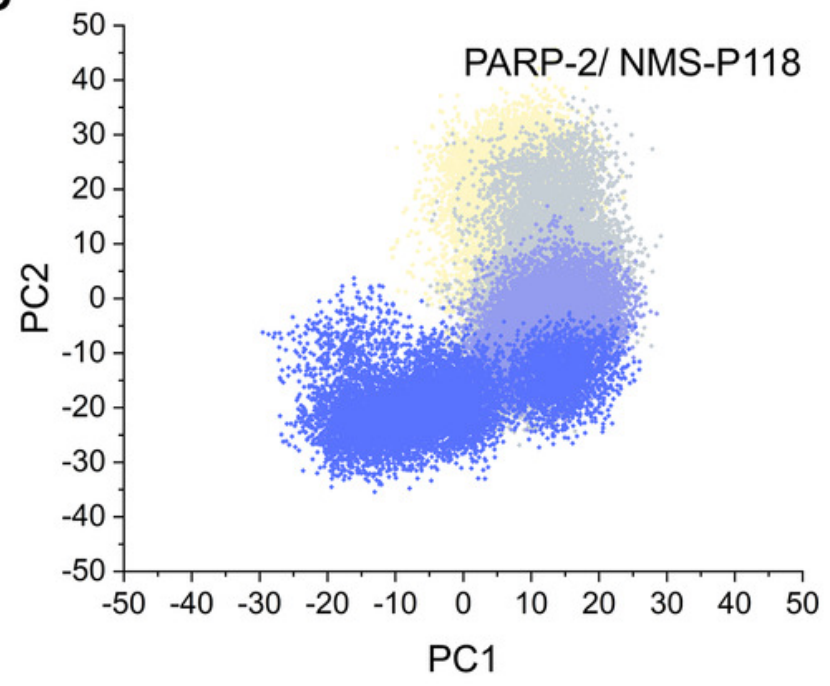

$\mathrm{D}$

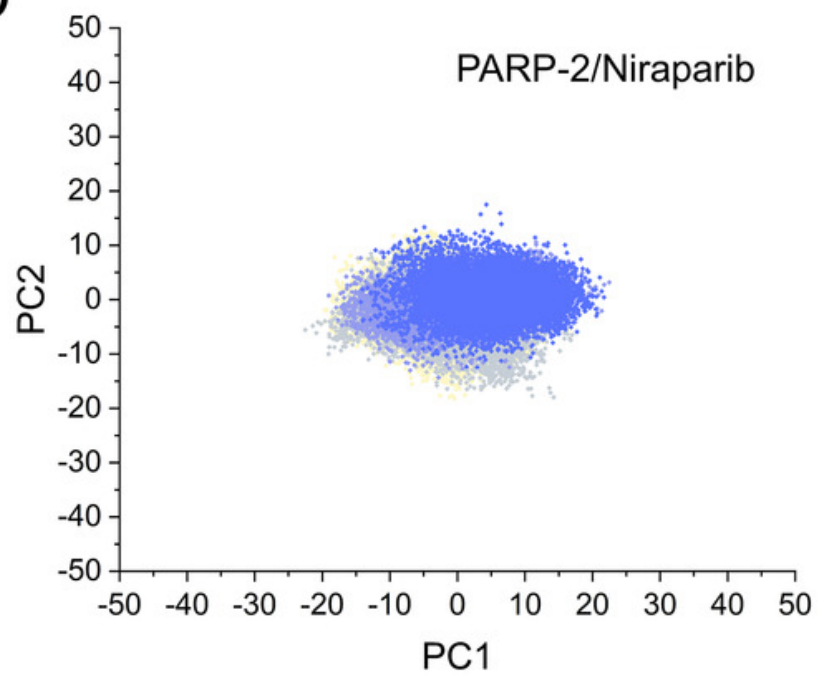

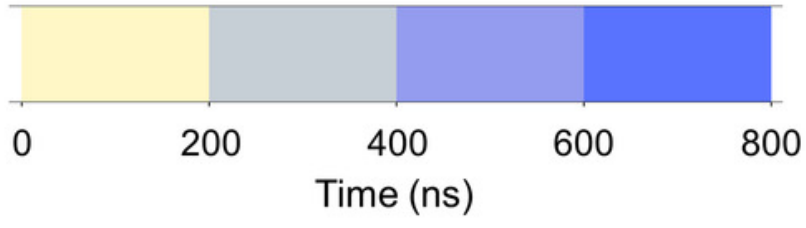


Figure 5

DCC analysis from the 800 ns classical MD simulations.

(A) PARP-1/NMS-P118. (B) PARP-2/NMS-P118. (C) PARP-1/Niraparib. (D) PARP-2/Niraparib.

A

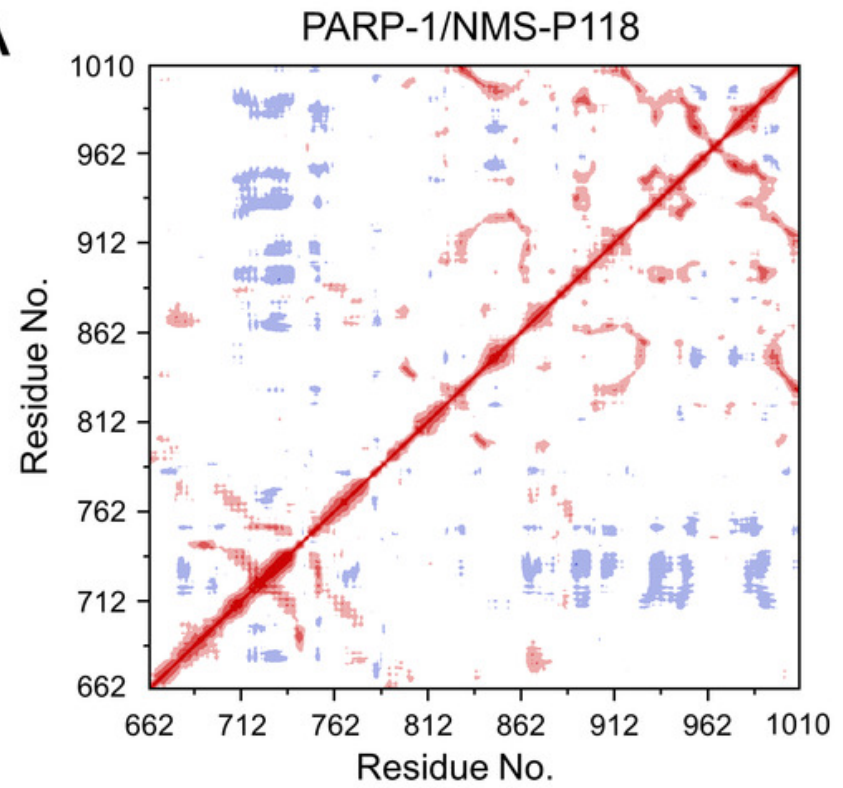

C

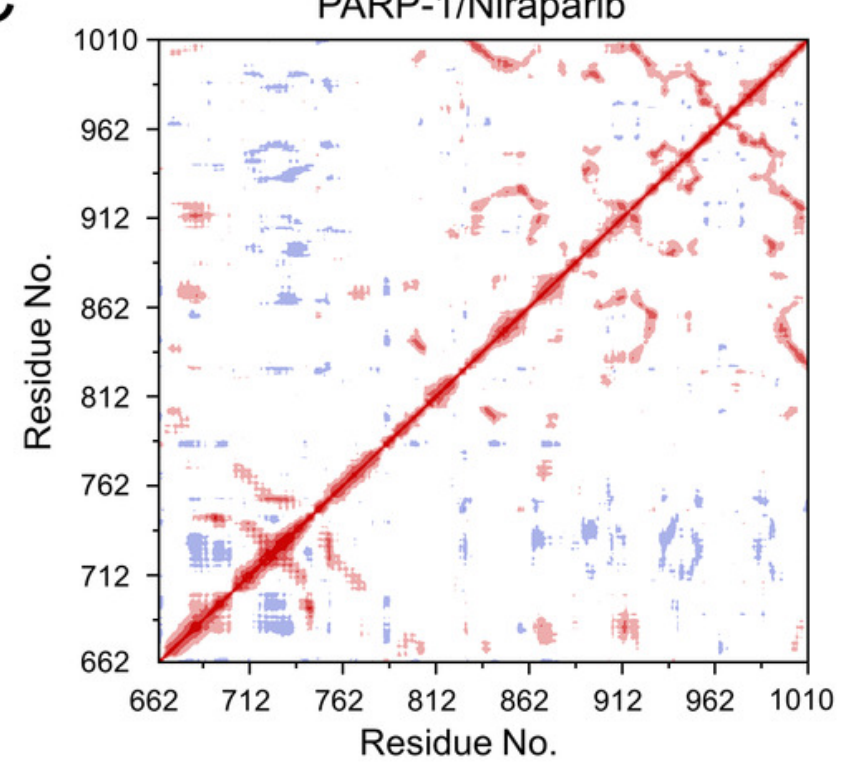

$0.75 \sim 1.0$

$\square 0.25 \sim 0.50$

$\square .50 \sim 0.75$

\begin{tabular}{|l}
$\square$ \\
$\square$
\end{tabular}
B

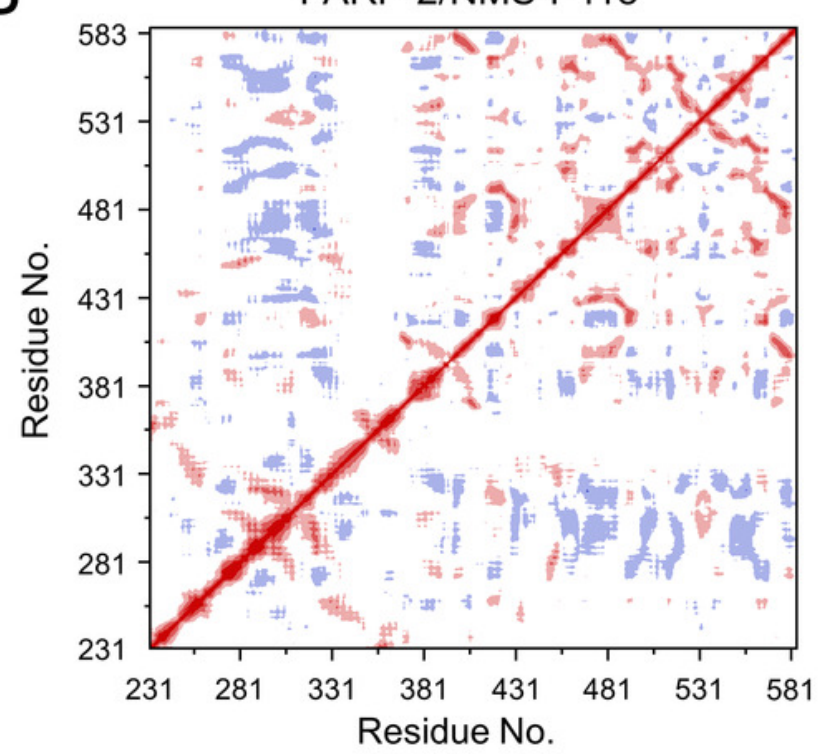

$D$

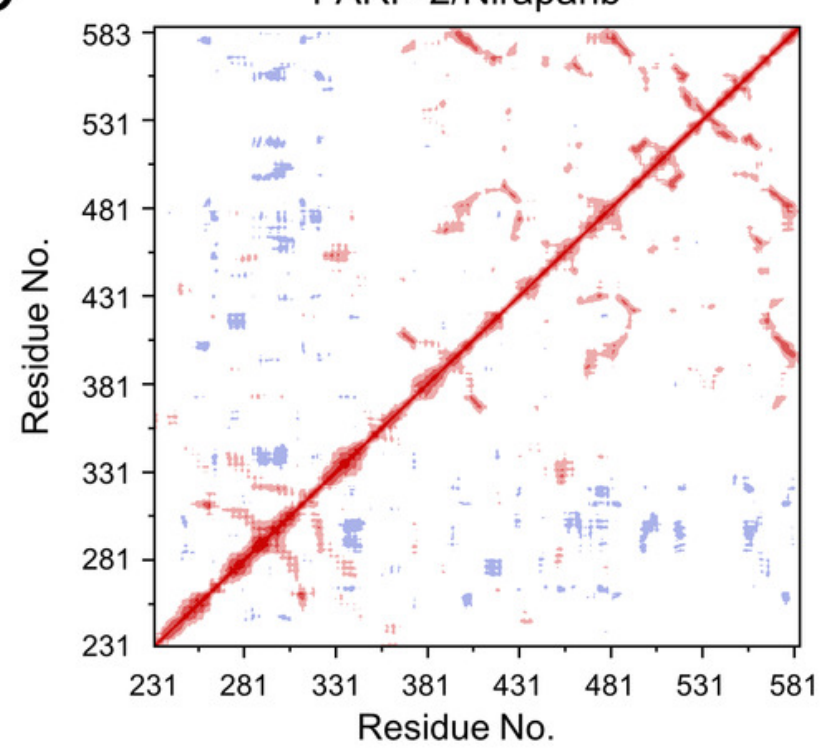

\begin{tabular}{|l}
$\square-0.50 \sim-0.25$ \\
\hline
\end{tabular}

$-1.0 \sim-0.75$ 
Figure 6

RMSF analysis from the 800 ns classical MD simulations.

(A) Alignment of RMSF of PARP-1/NMS-P118 and PARP-1/Niraparib. (B) Alignment of RMSF of PARP-2/NMS-P118 and PARP-2/Niraparib.
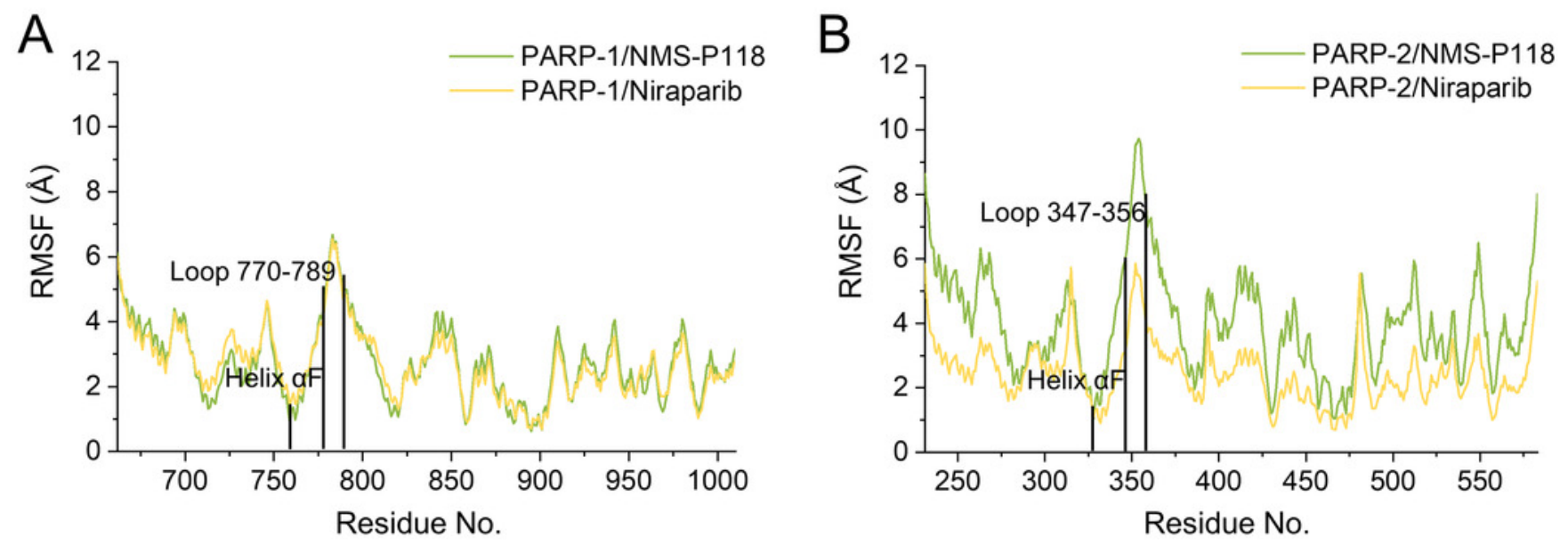
Figure 7

Energy difference in per-residue contributions between Niraparib and NMS-P118.

(A) The energy differences between PARP-1/NMS-P118 (green) and PARP-1/Niraparib (gray).

(B) Alignment of representative structures of PARP-1/NMS-P118 and PARP-1/Niraparib. (C) The energy differences between PARP-2/NMS-P118 and PARP-2/Niraparib. (D) Alignment of representative structures of PARP-2/NMS-P118 (green) and PARP-2/Niraparib (gray).

A

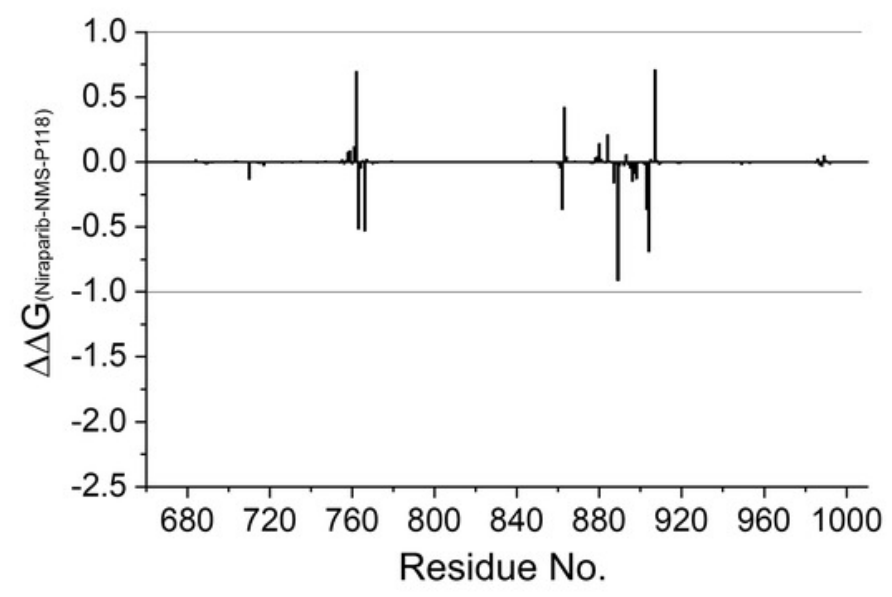

C

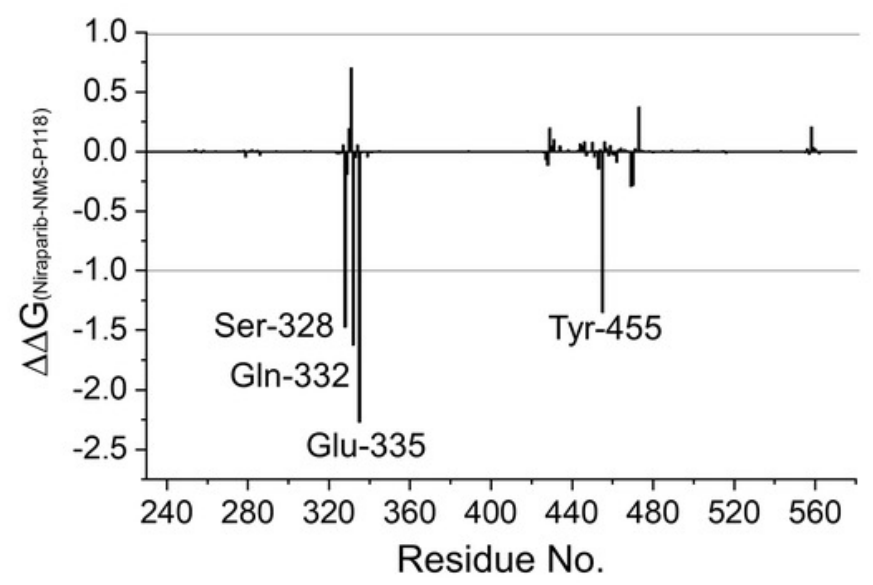

B

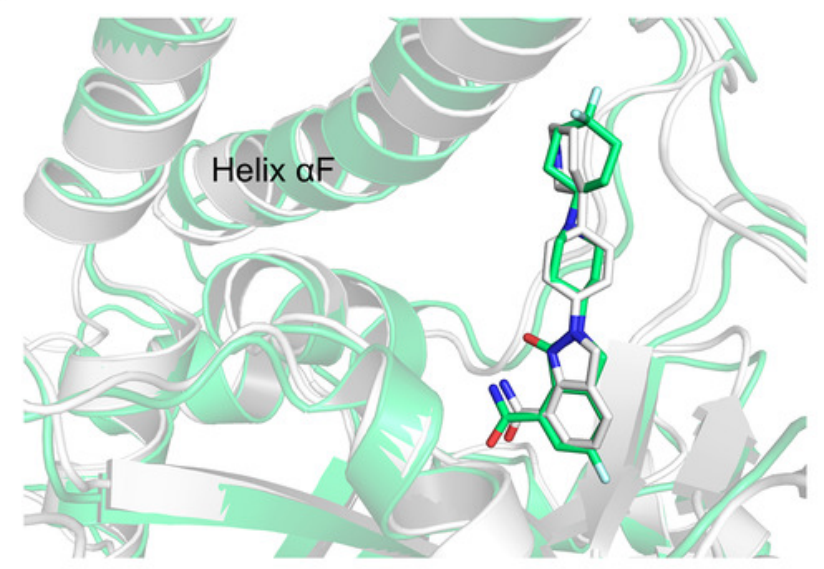

D

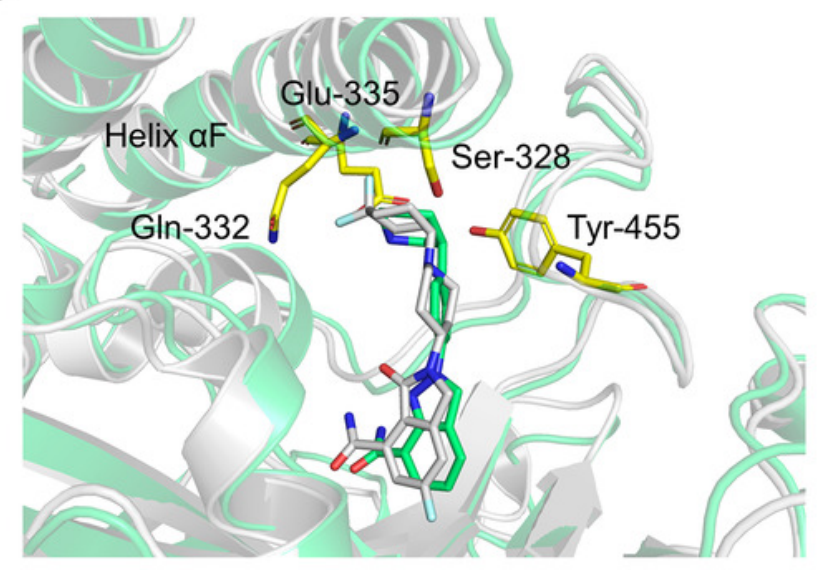


Figure 8

RMSD values of the protein backbones atoms and ligand heavy atoms from the $800 \mathrm{~ns}$ aMD simulations.

(A) PARP-1/NMS-P118. (B) PARP-2/NMS-P118. (C) PARP-1/Niraparib. (D) PARP-2/Niraparib.
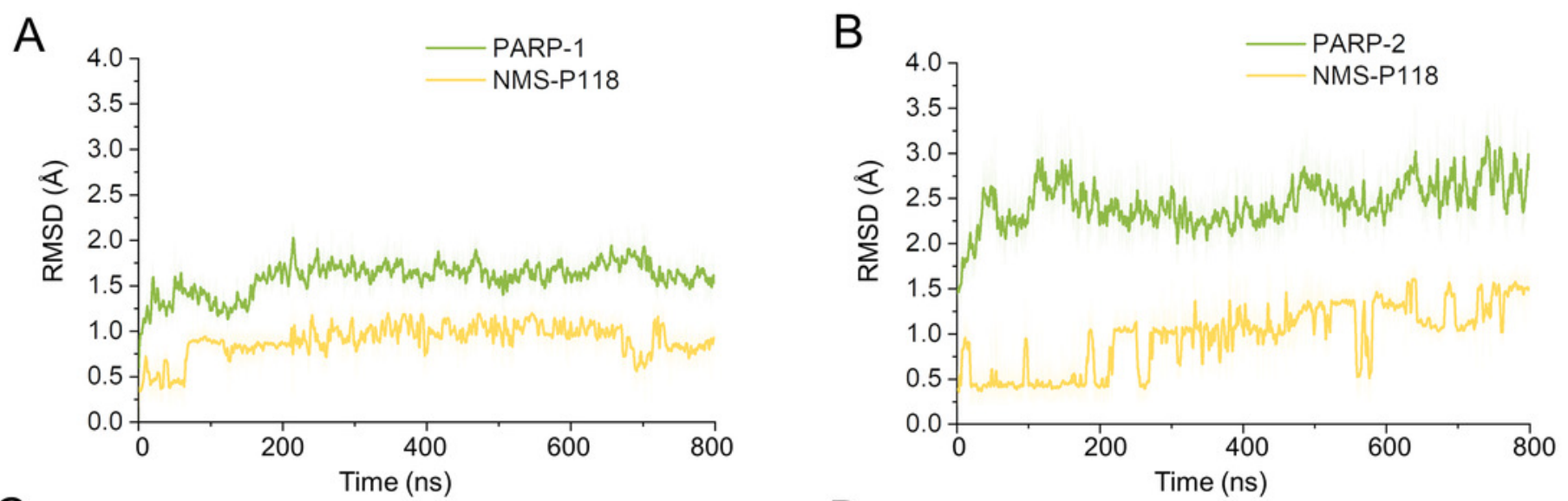

C
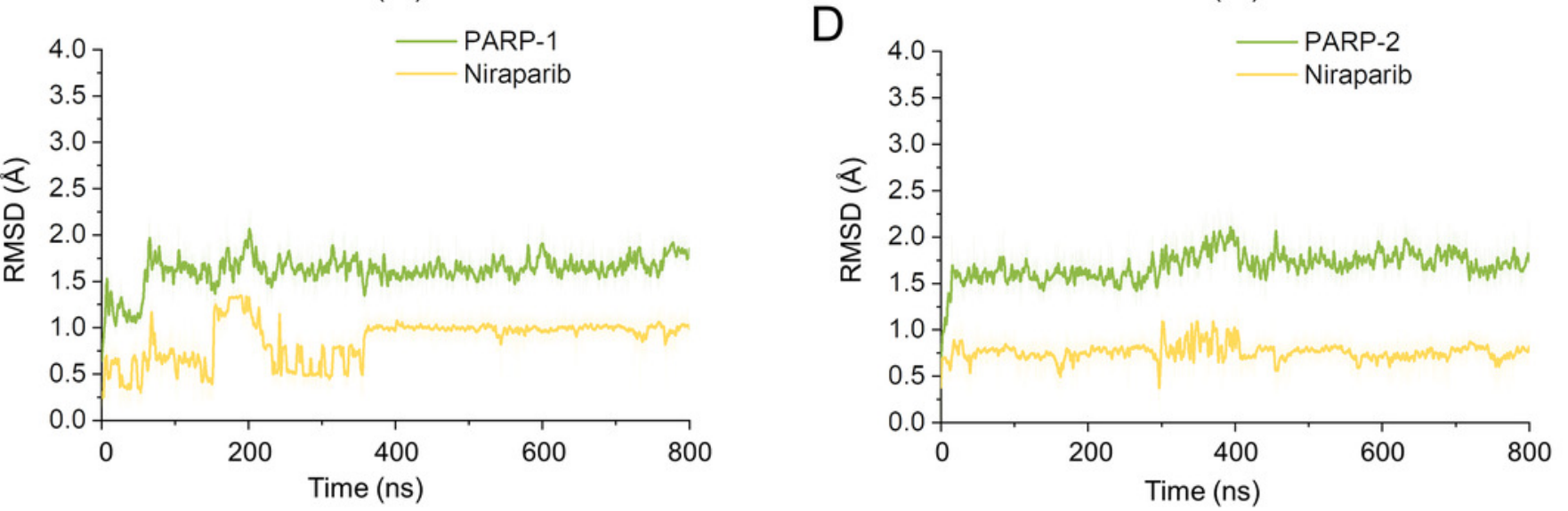
Figure 9

DCC analysis from the 800 ns aMD simulations.

(A) PARP-1/NMS-P118. (B) PARP-2/NMS-P118. (C) PARP-1/Niraparib. (D) PARP-2/Niraparib.

A

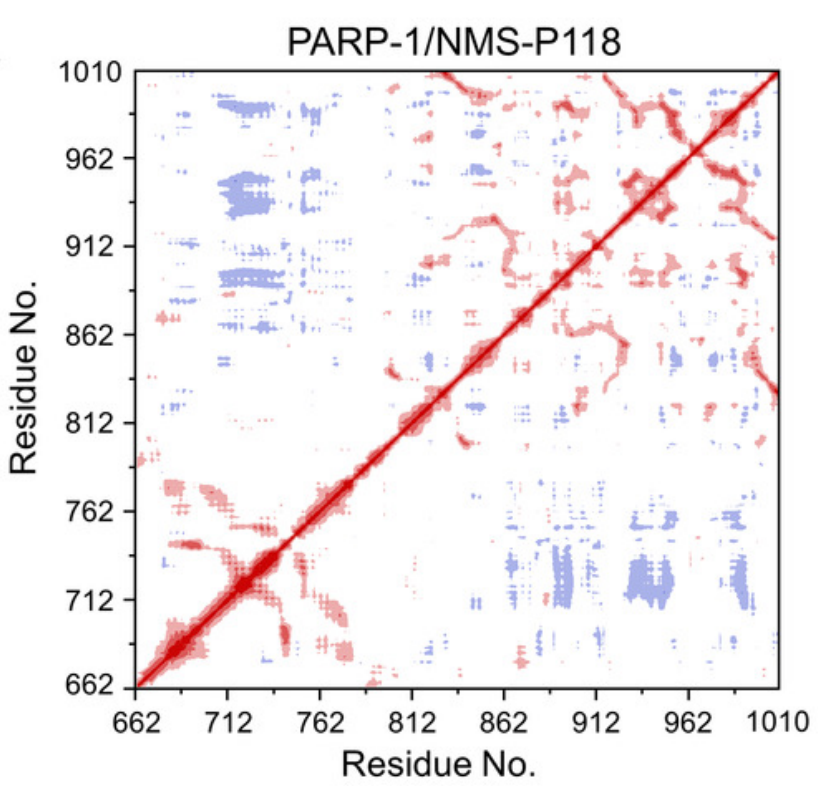

C

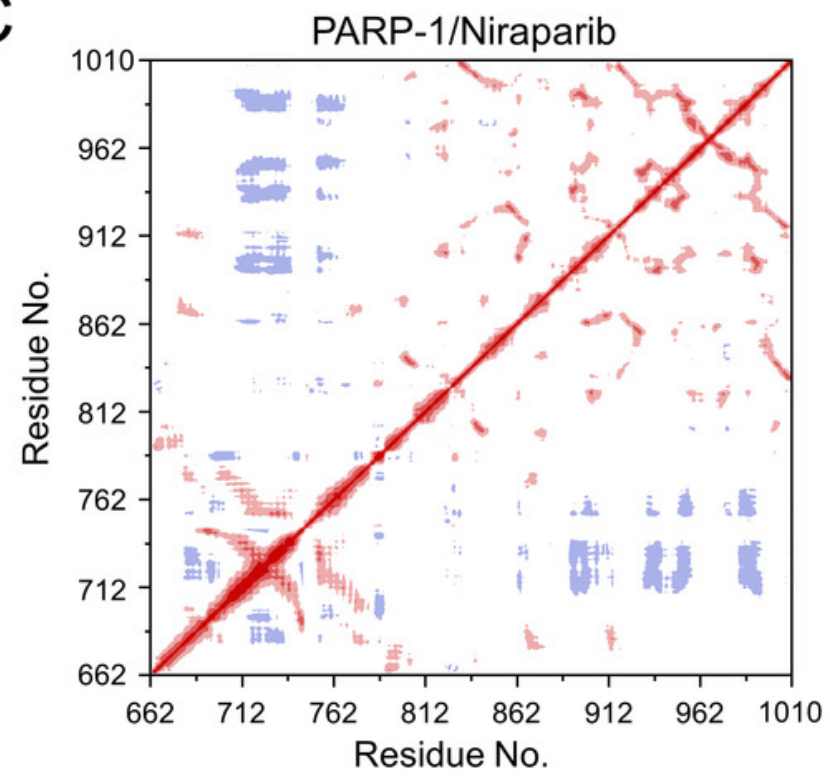

$0.75 \sim 1.0$

$0.25 \sim 0.50$

$\square 0.50 \sim 0.75$

$-0.25 \sim 0.25$
B

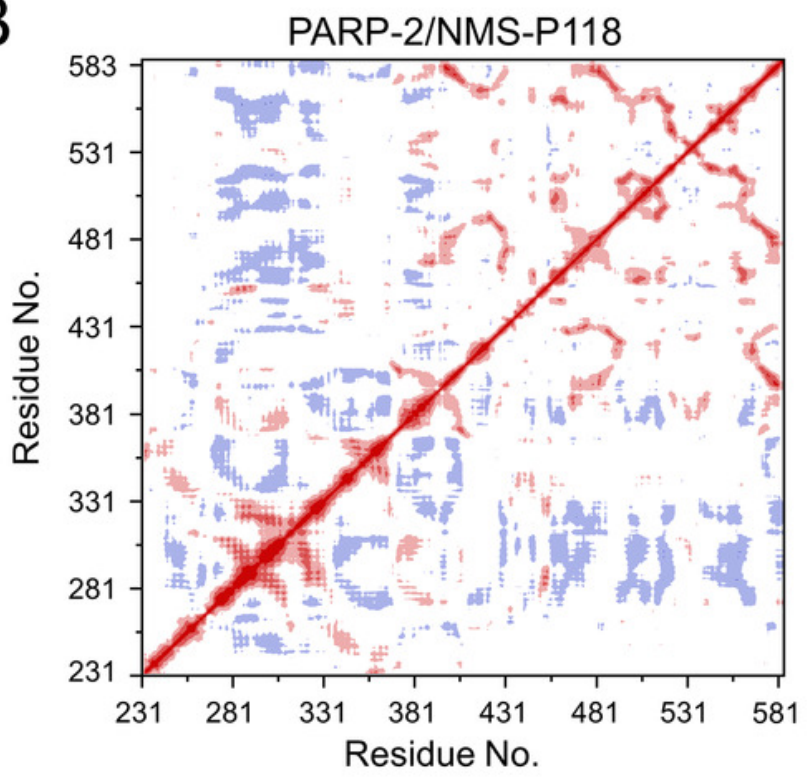

D

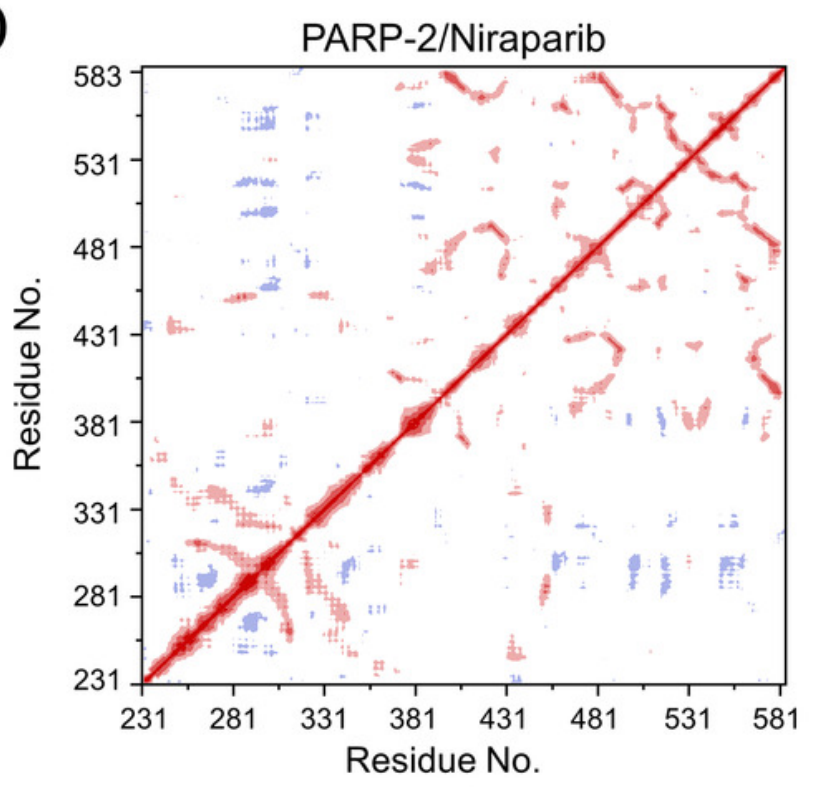

$-0.50 \sim-0.25$

$-1.0 \sim-0.75$ 
Figure 10

The top two ranked principal components (PC1, PC2) are plotted against each other from the 800 ns aMD simulations.

(A) PARP-1/NMS-P118. (B) PARP-2/NMS-P118. (C) PARP-1/Niraparib. (D) PARP-2/Niraparib.

A

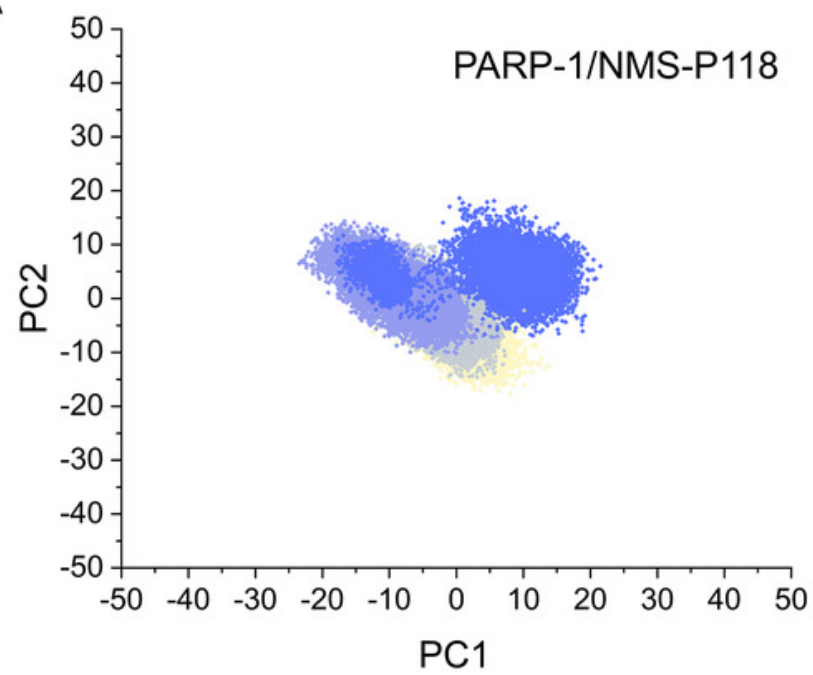

C

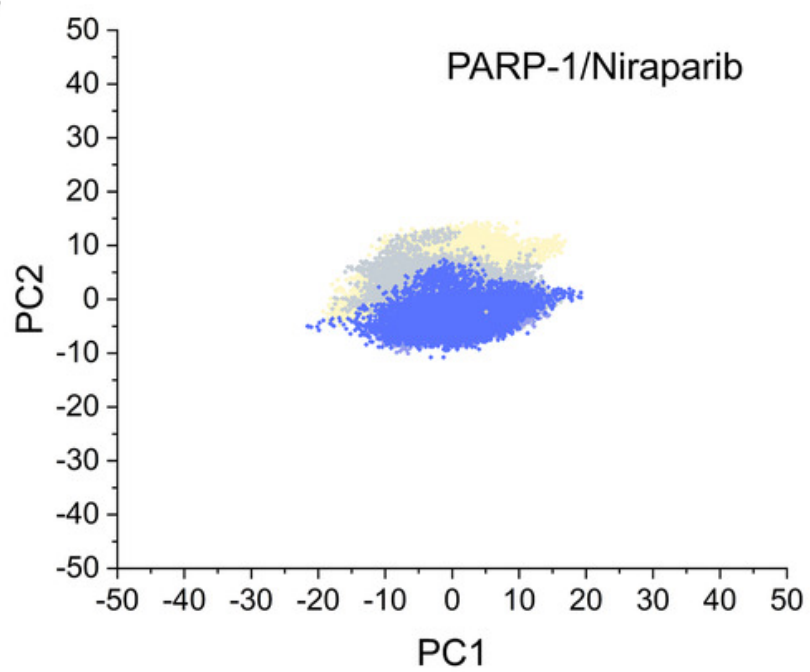

B

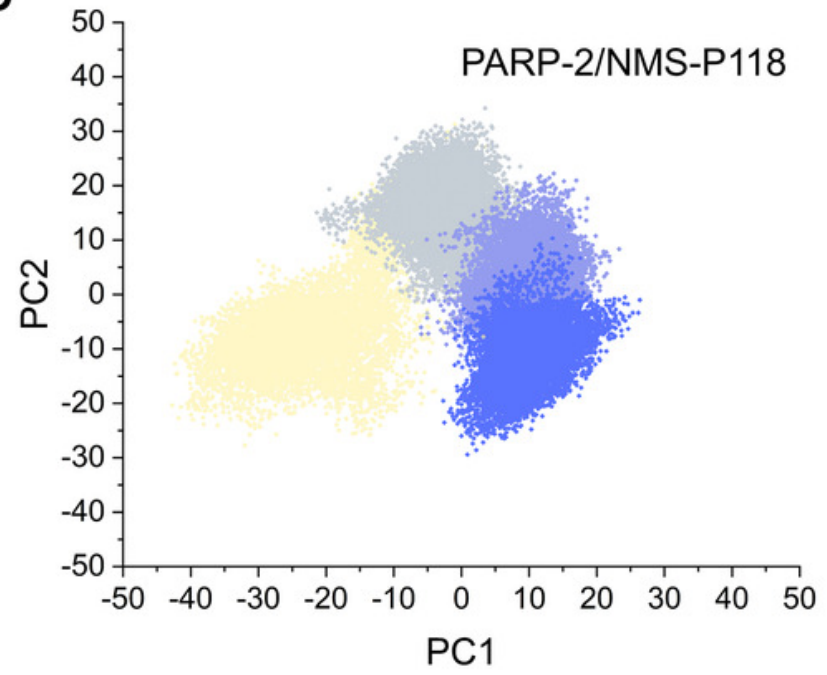

$\mathrm{D}$

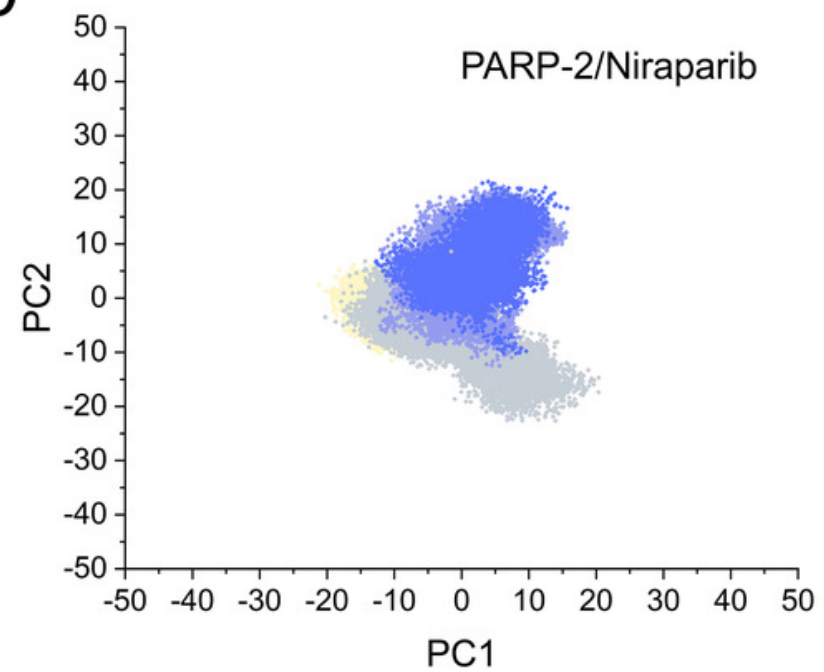

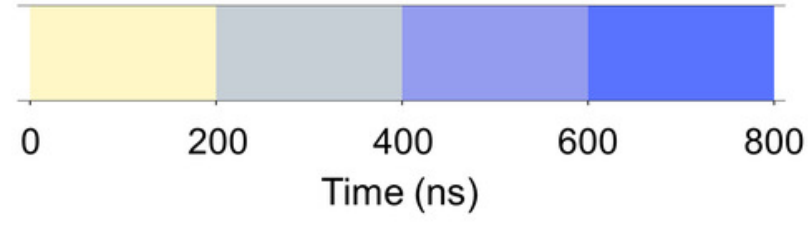


Figure 11

FEL analysis from the 800 ns aMD simulations.

(A) PARP-1/NMS-P118. (B) PARP-2/NMS-P118. (C) PARP-1/Niraparib. (D) PARP-2/Niraparib.

A

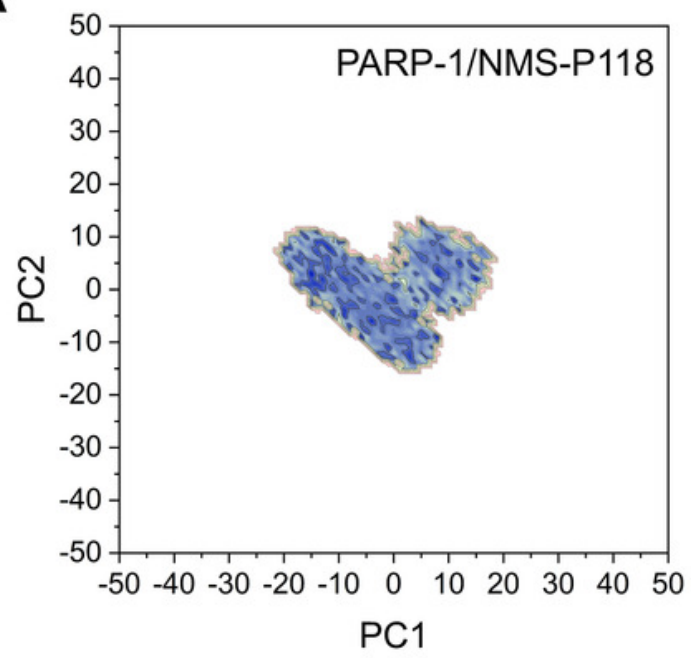

C

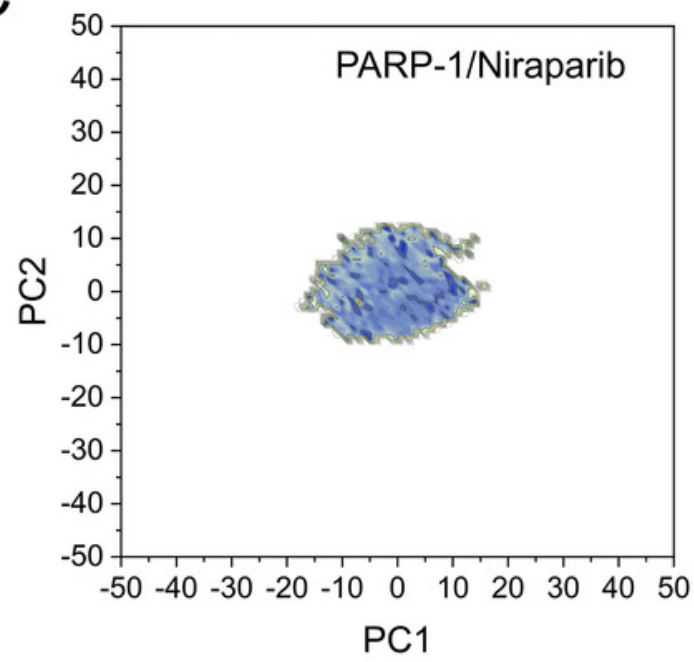

B

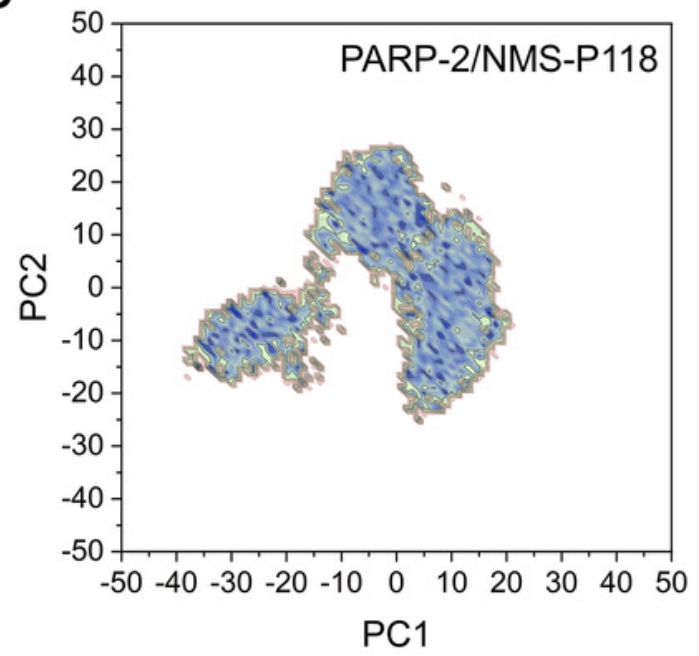

D

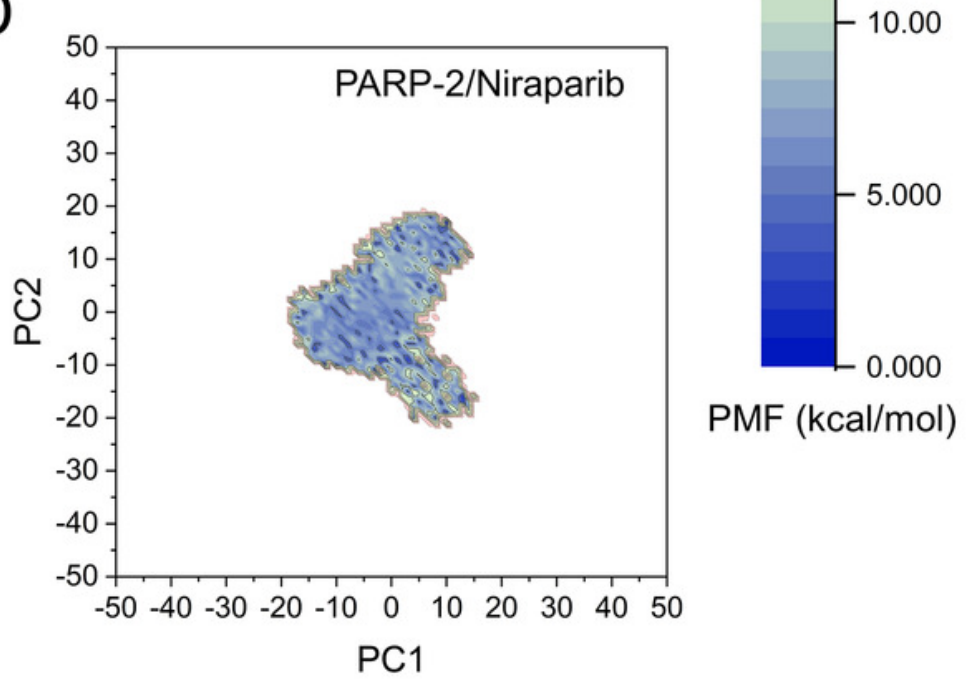

